\title{
A Gain-of-Function Screen Reveals Redundant ERF Transcription Factors Providing Opportunities for Resistance Breeding Toward the Vascular Fungal Pathogen Verticillium Iongisporum
}

\author{
Christian Fröschel, Tim Iven, Elisabeth Walper, Vanessa Bachmann, Christoph Weiste, \\ and Wolfgang Dröge-Laser ${ }^{\dagger}$
}

Department of Pharmaceutical Biology, Julius-von-Sachs-Institut, Biozentrum, Julius-Maximilians-Universität Würzburg, Würzburg, 97082, Germany

Accepted 8 March 2019.

\begin{abstract}
Verticillium longisporum is a vascular fungal pathogen leading to severe crop loss, particular in oilseed rape. Transcription factors (TF) are highly suited for genetic engineering of pathogen-resistant crops, as they control sets of functionally associated genes. Applying the AtTORF-Ex (Arabidopsis thaliana transcription factor open reading frame expression) collection, a simple and robust screen of TF-overexpressing plants was established displaying reduced fungal colonization. Distinct members of the large ethylene response factor (ERF) family, namely ERF96 and the six highly related subgroup IXb members ERF102 to ERF107, were identified. Whereas overexpression of these ERF significantly reduces fungal propagation, single loss-of-function approaches did not reveal altered susceptibility. Hence, this gain-of-function approach is particularly suited to identify redundant family members. Expression analyses disclosed distinct $E R F$ gene activation patterns in roots and leaves, suggesting functional differences. Transcriptome studies performed on chemically induced ERF106 expression revealed an enrichment of genes involved in the biosynthesis of antimicrobial indole glucosinolates (IG), such as CYP81F2 (CYTOCHROME P450-MONOOXYGENASE 81F2), which is directly regulated by IXb-ERF via two GCC-like
\end{abstract}

\section{Fröschel and T. Iven contributed equally to this article.}

Sequence data is available under the following accession numbers: ACTIN8 (At1g49240); CYP81F2 (At5g57220); ERF95 (At3g23220); ERF96 (At5g43410); ERF97 (At1g04370); ERF102 (At5g47230); ERF103 (At4g17490); ERF104 (At5g61600); ERF105 (At5g51190); ERF106 (At5g07580); ERF107 (At5g61590); MYB51 (At1g18570); PDF1.2A (At5g44420); UBQ5 (At3g62250).

Current address for Tim Iven: KWS SAAT SE, Postfach 14 63, 37555 Einbeck, Germany

${ }^{\dagger}$ Corresponding author: W. Dröge-Laser;

wolfgang.droege-laser@uni-wuerzburg.de

Funding: This work was supported by DFG grant numbers DR273/8-1, DR273/15-1.

*The $\boldsymbol{e}$-Xtra logo stands for "electronic extra" and indicates that six supplementary figures and four supplementary tables are published online.

The author(s) declare there is no conflict of interest.

๑) 2019 The American Phytopathological Society cis-elements. The impact of IG in restricting fungal propagation was further supported as the cyp81f2 mutant displayed significantly enhanced susceptibility. Taken together, this proofof-concept approach provides a novel strategy to identify candidate $\mathrm{TF}$ that are valuable genetic resources for engineering or breeding pathogen-resistant crop plants.

Keywords: defense signaling pathways, fungus-plant interactions, phytoalexins and secondary metabolism, plant antifungal responses, plant biotechnology

Transcription factors (TF) control sets of functionally associated genes and, therefore, are highly suited to be used for engineering stress-resistant crops. Plant genomes encode for numerous TF, highlighting the importance of transcriptional regulation. Currently more than $2,400 \mathrm{TF}$ are assigned to the model plant Arabidopsis thaliana (Guo et al. 2005; PérezRodríguez et al. 2010; Pruneda-Paz et al. 2014; Ramirez and Basu 2009; Yilmaz et al. 2011). Due to their conserved DNAbinding domains, plant TF can be organized in gene families that frequently consist of more than 100 members, having specified their functions during evolution. Hence, screening approaches are required to identify the candidate TF that are most suitable for crop engineering (Wehner et al. 2011b). In this study, we focused on the APETALA 2/ethylene response factor (AP2/ERF) family, which harbors several TF implicated in defense responses to various pathogens.

In Arabidopsis, the AP2/ERF superfamily consists of three distinct families, namely AP2, RAV, and ERF (Licausi et al. 2013; Nakano et al. 2006). All of these proteins are characterized by at least one AP2/ERF DNA-binding domain (Allen et al. 1998). Based on amino acid homology, the 121 ERF members have been classified into 12 groups. Six of these groups form a phylogenetically distinct branch, designated dehydration responsive element-binding (DREB) proteins. Whereas DREB generally bind A/GCCGAC elements in vitro, the other ERF are proposed to bind GCC boxes (AGCCGCC) (Licausi et al. 2013; Nakano et al. 2006; Ohme-Takagi and Shinshi 1995). Although DREB are frequently involved in abiotic stress signaling and several ERF mediate pathogen defense responses, a simple functional assignment is not possible (Huang et al. 2016). Particular members of the ERF subgroup IX (ERF91 to ERF107) (Nakano et al. 2006) have been well described as mounting resistance to various pathogens. 
Whereas ERF92 (ERF1), ERF94 (ORA59, octadecanoidresponsive Arabidopsis AP2/ERF59), and ERF96 are involved in JA (jasmonic acid) and ET (ethylene) mediated pathogen defense (Berrocal-Lobo et al. 2002; Catinot et al. 2015; Pré et al. 2008), ERF102 and ERF103 (AtERF5/6), as well as ERF104, are downstream targets of MAP3 and MAP6 signaling (Bethke et al. 2009; Meng et al. 2013; Xu et al. 2016). However, knowledge of particular functional differences and redundancies, as well precise target genes, is still limited within the 17 group IX members. More generally, functional analyses of ERF are hampered by their large numbers, their highly related DNA-binding properties, and functional redundancies. Hence, the impact of ERF is frequently not disclosed by lossof-function approaches. We, therefore, propose a gain-offunction screen to identify ERF involved in defense. Because these TF have not been analyzed with respect to defense against vascular fungi, we studied their responses to Verticillium longisporum.

$V$. longisporum is a soilborne fungus belonging to the Ascomycota (Depotter et al. 2016). By colonizing the plant xylem, vascular pathogens, such as Verticillium spp. or Fusarium spp. display a very specific life cycle (Berrocal-Lobo and Molina 2008; Depotter et al. 2016). Although the $V$. longisporum host range is limited to the Brassicaceae family, it is a serious threat to oilseed rape, which is of particular economic importance (Depotter et al. 2016; Johansson et al. 2006). The life cycle initiates from resting forms, termed microsclerotia, which survive long periods in soil and germinate in response to plant exudates. After germination, fungal hyphae colonize the root surface and penetrate the epidermal cell layer without forming appressoria (Eynck et al. 2007). The hyphae traverse the root cortex by intracellular and intercellular growth and enter xylem elements. At this stage, the fungus exhibits a biotrophic life style. Conidia, which bud from the hyphae, are carried with the transpiration stream and invade upstream vessels. After reaching the foliage, premature senescence is triggered by manipulating the cytokinin homeostasis of the plant (Reusche et al. 2013). Phenotypic stunted growth and chlorosis are observed, while the fungus switches to a more saprophytic life style (Depotter et al. 2016; Eynck et al. 2007). The crop production of infected plants is strongly reduced, and because no efficient fungicides are available, farmers generally rely on resistance breeding (Depotter et al. 2016). Hence, novel strategies are required to define genetic determinants of resistance.

Arabidopsis can be used as host plants for $V$. longisporum by applying an up-rooting infection method (Iven et al. 2012). For this model system, a generally applicable and simple screening procedure evaluating $\mathrm{TF}$-overexpressing (OE) plant lines for fungal colonization of the stem was established. This approach is based on the AtTORF-Ex (Arabidopsis thaliana TF open reading frame expression) resource, a large collection of transgenic lines expressing TF genes under control of the $35 \mathrm{~S}$ promoter (Wehner et al. 2011b; Weiste et al. 2007). Making use of GATEWAY-compatible TF ORF collections, sets of 30 to 50 TF genes were recombined simultaneously in plant transformation vectors to generate transgenic seed pools via Agrobacterium-mediated transformation. These seed pools of TF $\mathrm{OE}$ plants were evaluated for enhanced resistance to $V$. longisporum. From selected transformants, the respective TF transgene can easily be determined by PCR. As a proof-ofprinciple, AtTORF-Ex collections covering most of the ERF family members were screened, whereby a subgroup of closely related ERF was found to specifically exhibit a sustainable, enhanced resistance to $V$. longisporum. Moreover, we studied $E R F$ gene regulation and identified $C Y P 81 F 2$ (CYTOCHROME P450-MONOOXYGENASE 81F2) as a direct target gene that is involved in the production of antimicrobial indole glucosinolates (IG) (Bednarek et al. 2009; Clay et al. 2009). Along this line, mutation of $C Y P 81 F 2$ increased susceptibility to $V$. longisporum, suggesting ERF-induced IG as an efficient mechanism of Brassicaceae resistance to $V$. longisporum. Taken together, the isolated $E R F$ genes provide a valuable genetic resource for future crop improvement.

\section{RESULTS}

\section{Establishment of a procedure to screen infected} Arabidopsis plants for colonization by $\boldsymbol{V}$. longisporum.

A. thaliana can be readily infected by V. longisporum (V143) using an up-rooting method. In short, roots of 3-week-old plants are excavated from a sand and soil cultivation substrate, gently washed, and dipped in a V. longisporum spore solution. Several days after transferring the plants back to soil, fungal progression to the stem and leaves leads to visible symptoms such as stunting, reduction in leaf size, formation of vascular striping patterns, and early senescence (Fig. 1A through C). To rapidly assess fungal colonization, stem segments of infected plants are cut 30 days postinoculation (dpi), surface-sterilized, and placed on growth media supporting outgrowth of fungal hyphae, which have colonized the interior vasculature. Susceptible and tolerant plants can be scored easily, as demonstrated in Figure 1D. Moreover, by applying a LUCIFERASE-labeled $V$. longisporum strain, we could confirm that the hyphae growing out of the stem segments originated from the strain initially used for infection (Fig. 1E). This simple screening procedure is suitable for testing a huge number of infected plant lines and for categorizing the degree of colonization in a semiquantitative manner.

\section{Screening of the AtTORF-Ex collection discloses ERF TF OE plants that display enhanced resistance to $V$. longisporum.}

For plant infection, the AtTORF-Ex resource, a collection of transgenic TF OE plant lines, was used. To dissect the impact of ERF in mediating resistance to $V$. longisporum, 1,288 transformants representing 90 independent ERF OE lines were screened to recover 41 plants showing enhanced resistance (Fig. 1D). Because the screening procedure was based on pools of transgenic ERF OE plants, the identity of the particular transgene in each selected plant had to be elucidated. Identification of the $E R F$ transgenes was performed by PCR using specific primers targeting the GATEWAY att sites used to generate the AtTORFEx pool (Weiste et al. 2007). The sequencing results of the PCRamplified transgenes obtained from the resistant transformants are summarized in Table 1.

The TF frequently identified in the screen were the most reliable candidates. In this respect, ERF96 (subgroup IXc) stood out, as it was found six times among the forty-one resistant transformants. Remarkably, candidate TF that led to enhanced resistance clustered particularly in the ERF branch, whereas ERF60 was the only DREB-branch member that was found three times (Fig. 1F). Overall, 17 of 41 resistant lines harbored group IX ERF (Table 1; Figure $1 \mathrm{~F}$ and $\mathrm{G}$ ). This is in line with previous reports demonstrating the impact of the group IX ERF, in particular, in mediating pathogen resistance (Huang et al. 2016). It is also important to note, that ERF mediating resistance to $V$. longisporum clustered among subgroups of highly related family members, such as ERF104 to ERF107 (subgroup IXb) or ERF84 to ERF87 (group VIII). Hence, this gain-of-function approach is particularly suited to identify sets of functionally redundant TF members. In the following analyses, we focused on the most frequently found ERF members identified in subgroups IXb and IXc. 


\section{Overexpression of ERF from subgroups IXb and IXc} restricts propagation of $\boldsymbol{V}$. longisporum.

Two to three weeks after inoculating the roots, $V$. longisporum reaches the foliage due transmission of conidia via the xylem stream. To assess the impact of the identified ERF on resistance, $V$. longisporum DNA was quantitatively determined as a parameter of fungal propagation. Quantitative PCR (qPCR) was performed using rosette leaf material from wild-type (WT), ERF gain-of-function and loss-of-function plants at $21 \mathrm{dpi}$ (Fig. 2A). Due to potential redundancies, highly related subgroup members were also included in the study, particularly with respect to multiple loss-of-function approaches. In several independent experiments, overexpression of ERF96, ERF103, ERF104, $E R F 105$, and ERF106 resulted in significantly reduced fungal propagation. ERF107 overexpression showed only a tendency toward enhanced resistance, presumably due to the low rate of overexpression (Fig. 2A).

To address whether ERF TF are essential to restrict $V$. longisporum growth, single and multiple T-DNA insertion, RNA interference (RNAi), and artificial micro-RNA (amiR) lines were analyzed by quantifying fungal DNA. The amiR approach was used in case the generation of double mutants was hampered by tandem gene organization. The molecular analyses of these lines is provided in Supplementary Figure S1, displaying only residual background expression. However, in all tested single mutant lines, propagation of $V$. longisporum was comparable to WT plants. Due to the high degree of homology it is most likely that several ERF may function redundantly in mounting an efficient defense. Nevertheless, a loss-of-function approach targeting three related subgroup IXc
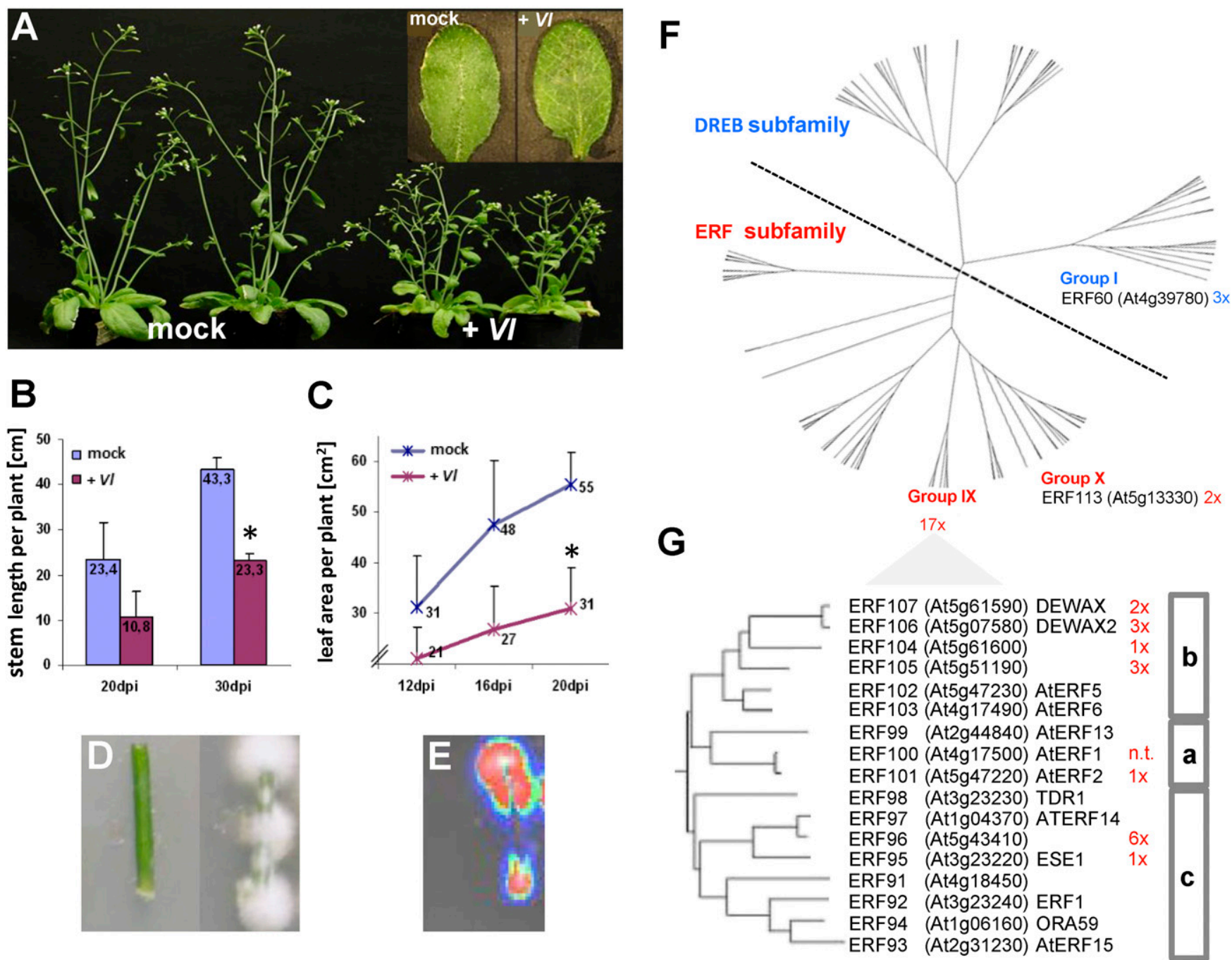

Fig. 1. Disease symptoms of Arabidopsis thaliana plants infected with Verticillium longisporum and results of screening of transcription factor (TF) overexpressors displaying enhanced resistance. A, Phenotype of A. thaliana Col-0 wild-type (WT) plants infected with $V$. longisporum (right) or mock-treated (left) at 28 days postinoculation (dpi). Inset: compared with mock treatment, infected leaves show typical vascular striping patterns. B, Quantification of stem length from mock-treated (blue) or V. longisporum-infected (purple) plants at the time points indicated. Mean values ( \pm standard deviation) are shown; $n=18$ plants for each treatment. C, Projected leaf area of mock-treated (blue) and V. longisporum-infected (purple) plants. Mean values ( \pm standard deviation) of three experiments are shown; $n=18$ plants for each treatment. Student's $t$ test was used to compare values with infected WT plants; * indicates $P \leq 0.05$. D, Phenotypic view of stems cut from an infected ethylene response factor $(E R F)$ gene-overexpressing line showing reduced colonization (left) and an infected Col-0 WT plant (right). Stems were cut at 30 dpi, surface-sterilized, and incubated on growth media. Fungal outgrowth became visible after 3 to 5 days. E, Luminograph of stems infected with a luciferase-tagged V. longisporum strain. F, Simplified phylogenetic tree of the ERF TF family, highlighting the dehydration responsive element-binding (DREB) protein (blue) and ERF (red) branch according to Nakano et al. (2006). The numbers summarize how often the corresponding ERF gene was found in the Arabidopsis thaliana transcription factor open reading frame expression (AtTORF-Ex) resource gain-of-function screen for enhanced resistance. In total, 1,288 ERF OE plants covering 90 independent ERF TF were analyzed, resulting in 41 resistant transformants. G, Detailed phylogenetic view of the group IX ERF, their systematic and common names, A. thaliana identifier, and further subgrouping (a to c) according to Nakano et al. (2006). The frequencies - how often a particular ERF was found in the screen —are highlighted (red). n.t. = not tested. 
genes (ERF95, ERF96, and ERF97) showed no increase in susceptibility compared with WT plants. With respect to group IXb, double mutants between the most related gene pairs were analyzed. Although the double mutant erf102/erf103 (also known as erf5erf6) (Meng et al. 2013) displayed a significantly higher susceptibility, V. longisporum proliferation in amiRerf104/erf105 and the erf106/erf107 double mutant was comparable to WT plants. Taken together, whereas overexpression of several ERF significantly enhanced resistance to $V$. longisporum, their functional impact, based on loss-of-function approaches, remains difficult to assess due to redundancy.

\section{Overexpression of ERF103 to ERF105 genes does not interfere with plant growth.}

To efficiently apply the identified $E R F$ genes in crop engineering, overexpression should not result in impairment of plant growth. Therefore, the projected leaf area of seven 21-day-old uninfected plants was analyzed for each line under investigation (Fig. 2B). Overexpression of ERF103, ERF104, and ERF105 and all loss-of-function lines showed no significantly altered growth compared with WT plants, while overexpression of ERF96, $E R F 106$, and ERF107 resulted in an approximately 40 to $50 \%$ reduction in projected leaf area. Representative phenotypes are presented in Figure 2C. These findings have important implications with respect to biotechnological applications.

Infection with $V$. longisporum specifically alters transcription of distinct subgroup IXb and IXc ERF genes.

Based on mining of public expression data sets, transcription of the $E R F$ genes investigated is regulated by various biotic and abiotic stress treatments (Supplementary Table S1). Transcript abundance of ERF102 to 104 increases in response to many biotic stress perturbations. Conversely, ERF106 and ERF107 are frequently downregulated by biotic stimuli but induced by hypoxia and dark treatments. Transcriptional responses of the $E R F$ genes upon $V$. longisporum infection may provide insights into their function in WT plants. Hence, transcript levels in $V$. longisporum-infected and mock-treated roots and leaves were compared (Fig. 3). With respect to expression analyses in roots, plants were grown and infected in a sterile in vitro system, avoiding influences from other microorganisms. Due to retarded infection of the foliage, transcriptional responses in leaves were studied up to 21 dpi using plants grown in a semisterile soil system. For the quantitative reverse transcription PCR (qRTPCR) analysis, the identified $E R F$ genes, as well as their closest relatives, were chosen. The subgroup IXc genes (ERF95, ERF96, and ERF97) were expressed at very low levels and induced in roots at 6 dpi (Fig. 3A). In line with this observation, these genes were hardly expressed in leaves and only moderately induced at late infection time points (21 dpi) (Fig. 3B; Supplementary Fig. S2). Hence, although leading to increased resistance when overexpressed, they are most likely not important players in the WT plant defense against $V$. longisporum.

In contrast to ERF104 and ERF107, which displayed a high basal transcript abundance, most subgroup IXb members showed moderate expression in mock-treated roots. Whereas ERF102, $E R F 103$, and ERF105 were induced in roots, ERF106 and ERF107 expression was reduced within 3 and 6 dpi (Fig. 3A). In leaves, the transcript abundance of all subgroup IXb genes was reduced up to $50 \%$ over the infection time course (Fig. 3B).

To confirm efficient infection in this experiment, fungal DNA was quantified in leaves (Fig. 3C). It should be noted that

Table 1. Frequencies of the Arabidopsis thaliana transcription factor open reading frame expression (AtTORF-Ex) resource lines selected for resistance to Verticillium longisporum ${ }^{\mathrm{a}}$

\begin{tabular}{|c|c|c|c|c|}
\hline \multirow[b]{2}{*}{ Resistant line frequency } & \multirow[b]{2}{*}{ Resistant line $A$. thaliana identifier } & \multicolumn{2}{|c|}{ Nomenclature $^{\mathrm{b}}$} & \multirow[b]{2}{*}{ Common name, TF, other name } \\
\hline & & AtERF No. & Group & \\
\hline \multicolumn{5}{|l|}{ Ethylene response factor } \\
\hline $1 \times$ & $5 \mathrm{~g} 47220$ & 101 & IXa & AtERF-2 \\
\hline $1 \times$ & $5 \mathrm{~g} 61600$ & 104 & $\mathrm{IXb}$ & \\
\hline $3 x$ & $5 \mathrm{~g} 51190$ & 105 & $\mathrm{IXb}$ & \\
\hline $3 x$ & $5 \mathrm{~g} 07580$ & 106 & $\mathrm{IXb}$ & DEWAX2 \\
\hline $2 x$ & $5 \mathrm{~g} 61590$ & 107 & $\mathrm{IXb}$ & DEWAX \\
\hline $1 \times$ & $3 g 23220$ & 095 & IXc & ESE1 \\
\hline $6 x$ & $5 \mathrm{~g} 43410$ & 096 & IXc & \\
\hline $1 \times$ & $3 g 20310$ & 083 & VIIIa & AtERF-7 \\
\hline $1 \times$ & $\lg 80580$ & 084 & VIIIb & \\
\hline $1 \times$ & $5 \mathrm{~g} 13910$ & 085 & VIIIb & LEP \\
\hline $1 \times$ & $5 \mathrm{~g} 18560$ & 086 & VIIIb & PUCHI \\
\hline $1 \times$ & $\lg 28160$ & 087 & VIIIb & AtERF-16 \\
\hline $1 \times$ & $3 \mathrm{~g} 61630$ & 067 & VI & CRF6 \\
\hline $1 \times$ & $4 \mathrm{~g} 23750$ & 064 & VI & CRF2 \\
\hline $1 \times$ & $3 \mathrm{~g} 16770$ & 072 & VIIa & EBP \\
\hline $1 \times$ & $1 \mathrm{~g} 72360$ & 073 & VIIa & HRE1 \\
\hline $2 \times$ & $5 \mathrm{~g} 13330$ & 113 & $\mathrm{Xa}$ & RAP2.6L \\
\hline \multicolumn{5}{|c|}{ Dehydration responsive element-binding protein } \\
\hline $1 \times$ & $5 \mathrm{~g} 52020$ & 025 & IIIb & \\
\hline $1 \times$ & $4 \mathrm{~g} 25480$ & 031 & IIIc & CBF3/DREB1A \\
\hline $1 \times$ & $4 \mathrm{~g} 32800$ & 043 & IIIe & \\
\hline $1 \times$ & $4 \mathrm{~g} 06746$ & 007 & IIa & PAP2.9 \\
\hline $1 \times$ & $4 \mathrm{~g} 36900$ & 009 & IIa & RAP2.10 \\
\hline $1 \times$ & $4 \mathrm{~g} 31060$ & 015 & $\mathrm{IIb}$ & \\
\hline $3 x$ & $4 \mathrm{~g} 39780$ & 060 & $\mathrm{Ib}$ & \\
\hline $1 \times$ & $5 \mathrm{~g} 65130$ & 057 & $\mathrm{Ib}$ & \\
\hline \multicolumn{5}{|l|}{ Double transformants ${ }^{\mathrm{c}}$} \\
\hline $1 \times$ & $2 \mathrm{~g} 40340+5 \mathrm{~g} 13910$ & $048+085$ & IVa+VIIIb & DREB2C+LEB \\
\hline $1 \times$ & $5 \mathrm{~g} 44210+3 \mathrm{~g} 60490$ & $035+080$ & IIId+VIIIb & AtERF-9 \\
\hline $1 \times$ & $4 \mathrm{~g} 25480+3 \mathrm{~g} 60490$ & $035+031$ & IIId+IIIc & CBF3 \\
\hline
\end{tabular}

${ }^{a}$ Forty-one plants were genotyped by PCR and subsequent DNA sequencing of the TF transgene as described by Weiste et al. (2007).

b Nomenclature groupings according to Nakano et al. (2006).

c Plants overexpressing two ERF genes. 
previous studies demonstrated posttranslational regulation by phosphorylation via MPK3/6 of ERF102, ERF103, and ERF104, which are well-known regulators in plant pathogen defenses (Bethke et al. 2009; Meng et al. 2013; Xu et al. 2016). Indeed, the crucial phosphorylation sites are conserved, in part, between ERF102 to ERF105. In contrast, ERF106 and ERF107 do not show these conserved sites (Supplementary Fig. S3), indicating that different regulatory mechanisms are involved.
Transcriptome studies reveal group IXb ERF to control pathogenesis-related $(P R)$ gene expression and biosynthesis of antimicrobial IG.

To identify potential ERF target genes, which are crucial for mediating resistance to $V$. longisporum., transcriptome studies were performed with 14-day-old XVE-ERF106 seedlings enabling estradiol (Est)-inducible expression of ERF106. Because Est-induced TF proteins become detectable by immuno-blotting

A Respective ERF transcript abundance in roots:
$1.0|1093|$
395
\begin{tabular}{l|l|l|}
2.5 & 577 & 90
\end{tabular}
$4.7|0.1| \begin{aligned} & 0.45 \\ & 0.09 \\ & 0.02\end{aligned} \mid$
0.3
$|0.005| 0.02 \mid$
\begin{tabular}{l|l}
0.2 & 0.01 \\
0.2 & 0.01
\end{tabular}

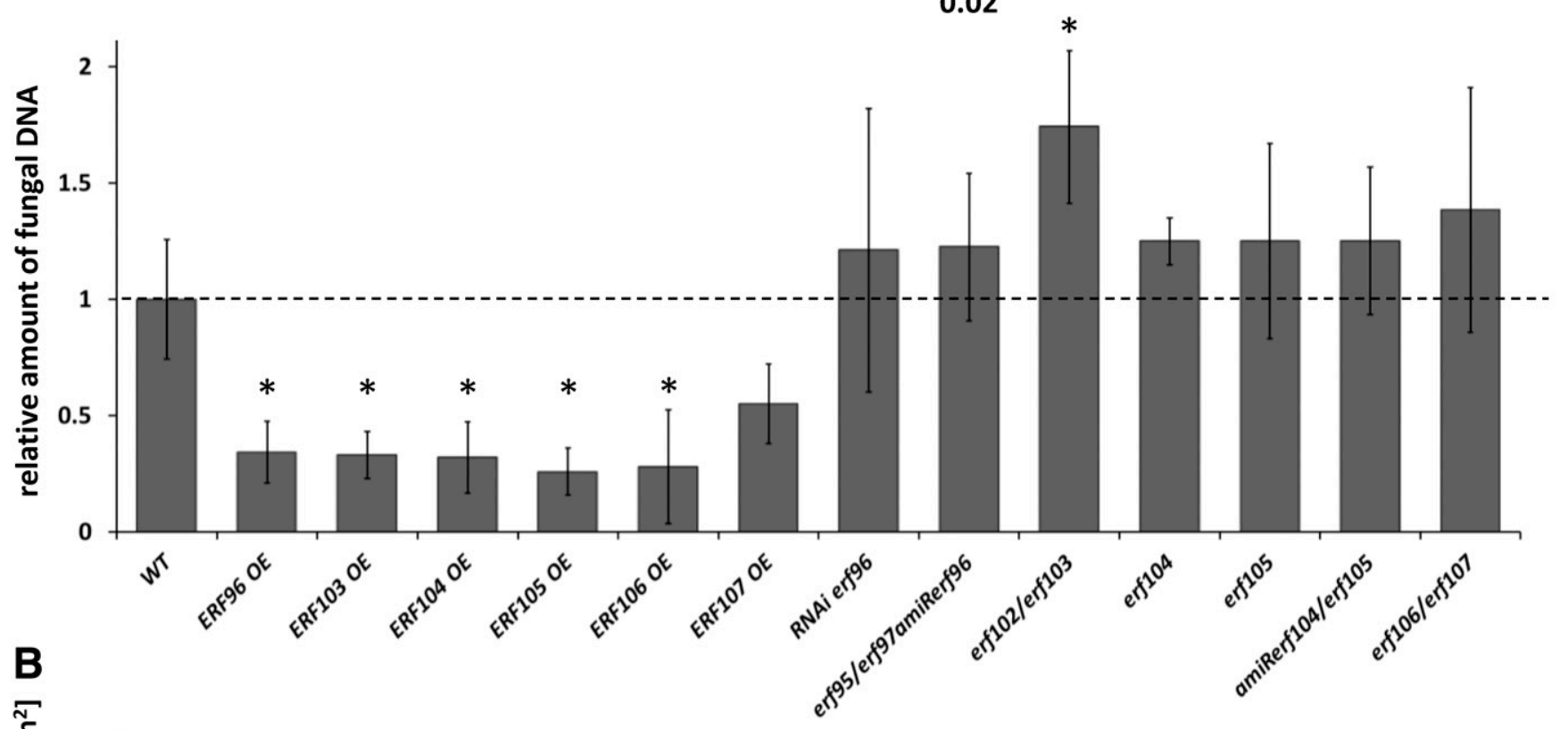

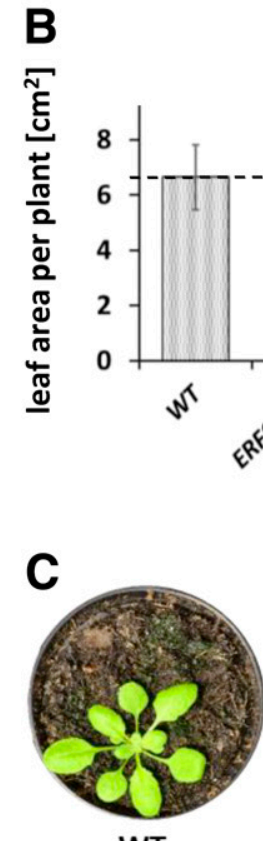

WT

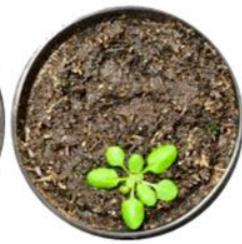

ERF96 OE

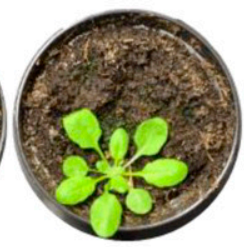

ERF103 OE
ERF104 OE

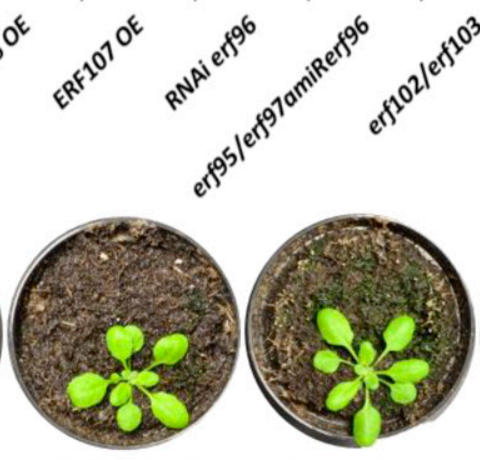

ERF105 OE

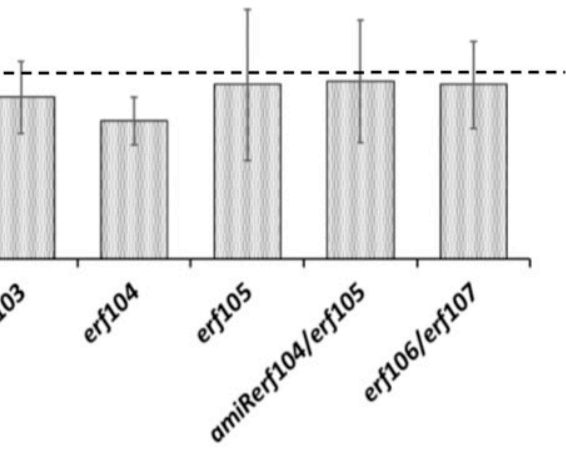

Fig. 2. Analysis of gain- and loss-of-function lines of ethylene response factor $(E R F)$ genes identified in the Arabidopsis thaliana transcription factor open reading frame expression (AtTORF-Ex) resource screen for enhanced resistance to Verticillium longisporum. A, Fungal DNA in leaf rosettes quantified by quantitative PCR (qPCR) at 21 days postinoculation (dpi). The relative amount of fungal DNA compared with wild-type (WT) plants is given for the ethylene response factor $(E R F)$ gene-overexpressing $(\mathrm{OE})$ plants and for single and multiple mutant approaches (erf [T-DNA insertion] RNA interference [RNAi]; artificial micro-RNA [amiR]). Values were normalized with respect to Arabidopsis ACTIN8 genomic DNA. Mean values ( \pm standard deviation) of fungal DNA relative to the infected WT plants (set to 1 ; dotted line) are shown; $n=3$ biological replicates, each consisting of the rosette leaves from five pooled plants. Student's $t$ test was used to compare values with infected WT plants; * indicates $P \leq 0.05$. Upper panel: $E R F$ transcript abundance as determined by quantitative reverse transcription PCR (qRT-PCR) in roots of gain- and loss-of-function plants relative to WT plants. For multiple mutants, values are given in the order they appear in the given names. B, Quantification of the projected green leaf area from 21-day-old uninfected plants given in A. Gain- and loss-of-function line WT plant level is indicated by a dotted line. Mean values ( \pm standard deviation) are shown; $n=7$ plants. Student's $t$ test was used to compare values with infected WT plants; ** indicates $P \leq 0.01$; *** indicates $P \leq 0.001$. C, Representative pictures of plants analyzed in part B. 
within 2 to $3 \mathrm{~h}$ (Walper et al. 2016; Weiste and Dröge-Laser 2014), RNA sequencing (RNAseq) experiments were performed after $4 \mathrm{~h}$ of induction to identify potential primary target genes (Supplementary Table S2). The efficiency of this approach was confirmed, as ERF106 transcripts were highly induced after Est treatment (Table 2). Notably, with respect to the other $E R F$ genes studied, only ERF103 showed a minor induction (log-fold change $[\log \mathrm{FC}]$ of 1.89$)$, indicating no or minor feedback regulation between the group IX ERF genes. Within the set of the strongest upregulated genes, many classical pathogenesis-related $(P R)$ genes, such as plant defensins $(P D F s)$, were found that are implicated in plant pathogen defense (Table 2) (Parisi et al. 2019). Moreover, many genes involved in the biosynthesis of tryptophan (Trp)-derived secondary compounds were
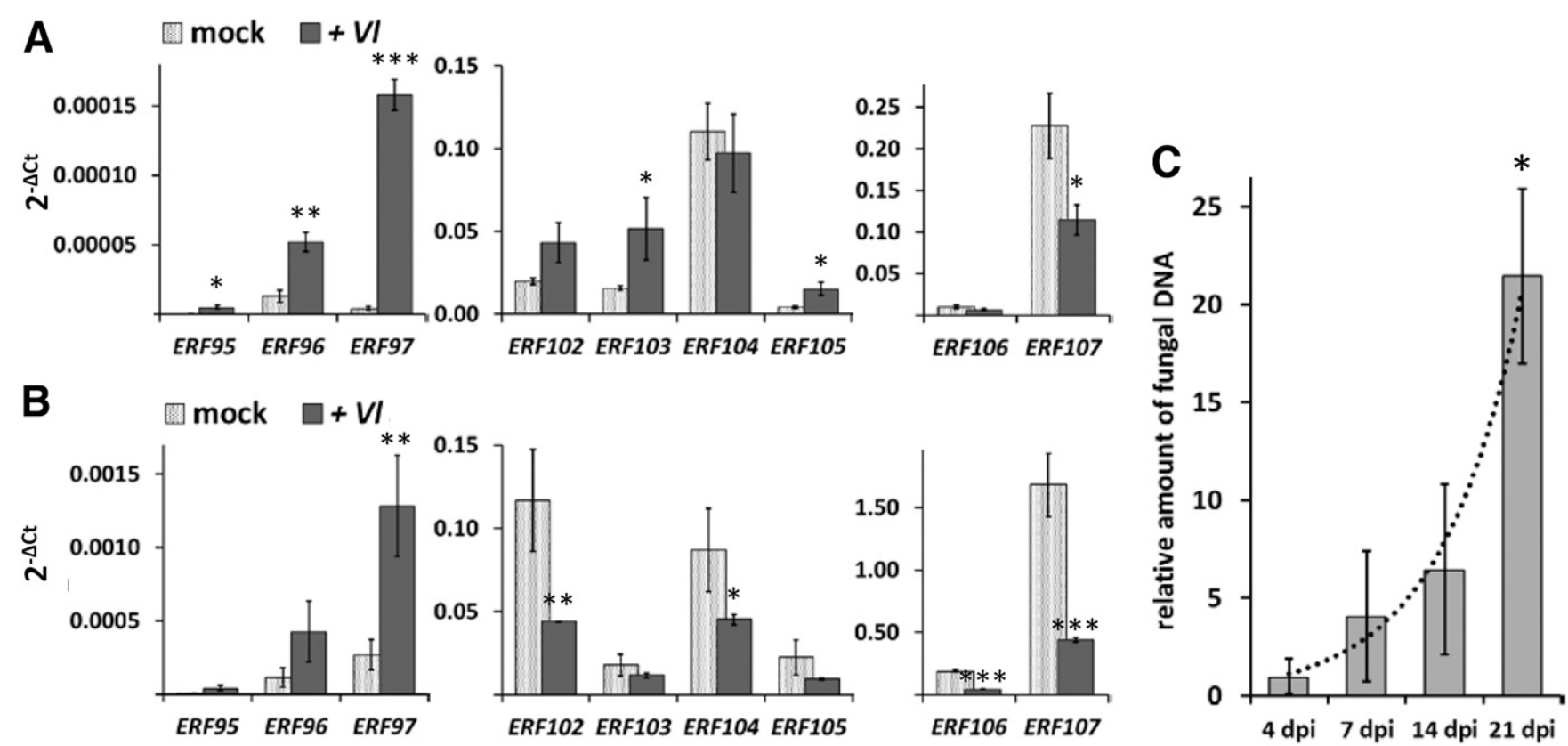

Fig. 3. Verticillium longisporum-induced transcriptional changes in the ethylene response factor $(E R F)$ genes analyzed in Arabidopsis thaliana roots and leaves. A, Quantitative reverse transcription PCR (qRT-PCR) analysis of roots grown on solid MS medium (in vitro root infection assay). ERF transcript abundance was analyzed in mock-treated and $V$. longisporum-infected plants at 6 days postinoculation (dpi). The scale of the y axis due to strong expressional differences is notable. B, qRT-PCR analysis of rosette leaves from infected plants grown on soil. $E R F$ transcript abundance was analyzed in mock-treated and $V$. longisporum-infected plants at $21 \mathrm{dpi}$. $2^{-\triangle \mathrm{Ct}}$ values were normalized to $U B Q 5$. Mean values ( \pm standard deviation) are shown; $n=3$ biological replicates. The scale of the y axis due to strong expressional differences is notable. Student's $t$ test was used to compare values with infected mock-treated plants; * indicates $P \leq 0.05$; ** indicates $P \leq 0.01$; *** indicates $P \leq 0.001$ (A and B). C, Successful infection was verified by quantification of fungal DNA in rosette leaves at 4,7 , 14, and 21 dpi. Mean values ( \pm standard deviation) were calculated after normalization to Arabidopsis ACTIN8 genomic DNA relative to the 4 dpi time point (set to 1$) ; n=3$ biological replicates for each time point. Student's $t$ test was used to compare values with $4 \mathrm{dpi} ; *$ indicates $P \leq 0.05$.

Table 2. Upregulated genes selected from a RNAseq experiment studying 14-day-old XVE-ERF106 seedlings

\begin{tabular}{|c|c|c|c|}
\hline Category & A. thaliana identifier & $\log \mathrm{FC}^{\mathrm{a}}$ & Gene product description \\
\hline \multirow{5}{*}{ Ethylene response factor genes } & $5 \mathrm{G} 07580$ & 6.51 & ERF106 \\
\hline & $1 \mathrm{G} 33760$ & 5.97 & ERF022 \\
\hline & 4G06746 & 4.97 & ERF007; DEAR5 \\
\hline & 4G17490 & 1.89 & ERF103; ethylene responsive element-binding factor 6 (AtERF6) \\
\hline & $1 \mathrm{G} 06160$ & 1.68 & Octadecanoid-responsive Arabidopsis AP2/ERF59 (ORA59) \\
\hline \multirow{6}{*}{$P R$ genes } & $2 \mathrm{G} 26010$ & 8.62 & Plant defensin 1.3 (PDF1.3) \\
\hline & $5 \mathrm{G} 44420$ & 7.11 & Plant defensin 1.2 (PDF1.2) \\
\hline & 2G14610 & 6.94 & Pathogenesis-related gene 1 (PR1) \\
\hline & $5 \mathrm{G} 44430$ & 5.87 & Plant defensin 1.2C (PDF1.2c) \\
\hline & $2 \mathrm{G} 26020$ & 5.72 & Plant defensin 1.2B (PDF1.2b) \\
\hline & $1 \mathrm{G} 75050$ & 4.15 & Thaumatin \\
\hline \multirow[t]{3}{*}{ Tryptophan biosynthesis } & 3G54640 & 3.48 & Tryptophan synthase alpha chain (TSA1) \\
\hline & $5 \mathrm{G} 05730$ & 2.23 & Anthranilate synthase alpha subunit 1 (ASA1) \\
\hline & $1 \mathrm{G} 25220$ & 1.99 & Anthranilate synthase beta subunit 1 (ASB1) \\
\hline \multirow[t]{8}{*}{ Tryptophan-derived indole glucosinolates } & $1 \mathrm{G} 18570$ & 2.18 & MYB domain protein 51 (MYB51) \\
\hline & 4G31500 & 2.30 & Cytochrome P450, family 83, subfamily B (CYP83B1); superroot 2 (SUR2) \\
\hline & 5G57220 & 4.46 & Cytochrome P450, family 81 , subfamily F (CYP81F2) \\
\hline & $1 \mathrm{G} 21100$ & 2.26 & Indole glucosinolate O-methyltransferase 1 (IGMT1) \\
\hline & $1 \mathrm{G} 21120$ & 2.19 & Indole glucosinolate O-methyltransferase 2 (IGMT2) \\
\hline & $1 \mathrm{G} 21110$ & 2.27 & Indole glucosinolate O-methyltransferase 3 (IGMT3) \\
\hline & $1 \mathrm{G} 21130$ & 2.44 & Indole glucosinolate O-methyltransferase 4 (IGMT4) \\
\hline & $1 \mathrm{G} 27130$ & 1.33 & Glutathione S-transferase tau 13 (GSTU13) \\
\hline \multirow[t]{2}{*}{ Camalexin biosynthesis } & 1G02930 & 2.31 & Glutathione S-transferase 6 (GSTF6) \\
\hline & $3 \mathrm{G} 26830$ & 1.84 & Phytoalexin deficient 3 (PAD3) \\
\hline
\end{tabular}

\footnotetext{
${ }^{\mathrm{a}}$ Log-fold change values after $4 \mathrm{~h}$ of estradiol induction relative to the untreated control $(P \leq 0.001)$.
} 
strongly induced after ERF106 overexpression. The primary amino acid Trp is shuttled into a complex network via two cytochrome P450 bottle-neck enzymes (CYP79B2 and CYB79B3) (Zhao et al. 2002), producing several secondary metabolites, such as camalexin or IG, that act as antimicrobial compounds against various pathogenic species (Ahuja et al. 2012; Frerigmann and Gigolashvili 2014). As shown in Table 2, genes involved in Trp biosynthesis (e.g., TSAI [TRYPTOPHAN SYNTHASE ALPHA CHAIN]) and generation of secondary antimicrobial IG metabolites were upregulated, such as CYP81F2 (CYTOCHROME-P450-MONOOXYGENASE $81 F 2$ ) or IGMT1/2 (INDOLE GLUCOSINOLATE OMETHYLTRANSFERASE 1/2) (Bednarek et al. 2009; Frerigmann and Gigolashvili 2014; Pfalz et al. 2011). In line with this observation, the gene encoding MYB51 (MYB-DOMAIN PROTEIN 51), a key TF in regulation of IG biosynthesis (Frerigmann and Gigolashvili 2014), was strongly induced by ERF106, as well as PAD3 (PHYTOALEXIN DEFICIENT 3), encoding a key enzyme in camalexin biosynthesis (Schuhegger et al. 2006). Trp-derived secondary metabolites have recently been described as supporting resistance to V. longisporum (Iven et al. 2012) and, hence, provide a likely mechanism enabling ERF106 and relatives to mediate resistance.

Public transcriptome data are available for overexpression approaches studying ERF96, ERF103, and ERF104 (Bethke et al. 2009; Catinot et al. 2015; Dubois et al. 2013; Meng et al. 2013). It should be noted that plant growth and experimental conditions differed considerably between these studies. Nevertheless, they complement the XVE-ERF106 transcriptome data. The upregulated genes found after Est-induced expression of ERF106, overexpression of ERF104 (p35S::ERF104) (Bethke et al. 2009; GEO accession GSE11807), or dexamethasoneinduced expression of ERF103 (ERF6 $\left.{ }^{I O E}-S\right)$ (Dubois et al. 2013) are compared in Figure 4A. These analyses demonstrate substantial gene regulatory specificity of the specific ERF, as well as partial redundancy. Within the eighteen commonly upregulated genes, a recognizably enriched number of four genes $(22 \%)$ are involved in IG biosynthesis. A Venn diagram including $E R F$ OE data sets after elicitation is provided in Supplementary Figure S4. Further pairwise comparisons of overexpression approaches are provided in Supplementary Figures S5 and S6.

Notably, genes involved in Trp-derived secondary metabolites are not upregulated in ERF96 OE plants, whereas expression of typical defense genes (PDF1.2A, PR4 [pathogenesis-related 4]) or pathogen-related TF genes (ORA59, WRKY18) were induced. Taken together, the gene regulatory capacities of ERF96 and group IXb-ERF differ considerably with respect to the biosynthesis of Trp-derived antimicrobial compounds.

\section{Via two GCC-like cis elements, group IXb ERFs directly activate the $C Y P 81 F 2$ gene, encoding a central enzyme in IG biosynthesis.}

Yet, only ERF103 has been demonstrated to bind the CYP81F2 promoter involved in IG biosynthesis $(\mathrm{Xu}$ et al. 2016). Therefore, whether the other ERF act accordingly was tested. Activation of a 1.6-kb promoter of the $C Y P 81 F 2$ gene fused to the GUS reporter was tested after coexpression of the ERF studied (Fig. 4B and C). ERF103 and ERF105 led to a sixfold induction of the reporter in protoplasts, whereas ERF96, ERF104, ERF106, and ERF107 activated the reporter by threefold. Overall, four GCC-like elements were detected in the $1.6-\mathrm{kb}$ promoter but not a perfect GCCGCC box. Single sequence-specific mutations in any of these sites did not abolish the gene activation capacities of ERF103 and ERF105 (Fig. 4D). However, a double mutation, in boxes 3 and 4 completely impaired promoter activation by ERF (Fig. 4E). A minor reduction in ERF-mediated reporter activation also was observed after mutation of boxes 1 and 2 . It should to be noted that endogenous proteins might bind and regulate the reporter. Mutation constructs, therefore, may result in lower background activities.

To confirm direct promoter binding, chromatin immunoprecipitation coupled to PCR $\left(\mathrm{ChIP}_{\mathrm{PCR}}\right)$ was performed using the strongest activator, ERF105, as a prototypic example (Fig. 4F). In accordance with the promoter mapping experiment in protoplasts, a direct, highly significant binding to boxes 3 and 4, but no binding to boxes 1 and 2, was observed. Due to limitations in shearing (major fragment sizes were approximately $500 \mathrm{bp}$ ), binding to closely adjacent boxes 3 and 4 could not further be resolved. Based on these results, we propose that there is a direct regulation of the CYP81F2 promoter by the ERF105-related group IXb TF.

\section{Overexpression of subgroup IXb ERF results}

in constitutive activation of IG pathway genes in planta.

The ability of the ERF OE lines studied to activate gene expression in uninfected plants was evaluated further. Based on the transcriptome results, $C Y P 81 F 2$ and $M Y B 51$ encoding for a pathogen-induced IG-specific biosynthetic gene and an IG transcriptional master regulator, respectively, were selected (Bednarek et al. 2009; Clay et al. 2009; Gigolashvili et al. 2007; Pfalz et al. 2009). Moreover, PDF1.2A was used to monitor a commonly regulated gene found to be activated by members of subgroups IXb and IXc (Huang et al. 2016; Zarei et al. 2011).

Although ERF96 OE plants showed a more than 1,000-fold increase in ERF96 transcript abundance compared with WT plants (Fig. 5A), only minor changes in CYP81F2 (Fig. 5B) transcripts were observed in roots or leaves. Hence, $C Y P 81 F 2$ is not considered a strong candidate for an ERF96 target gene. Nevertheless, significant activation of MYB51 (Fig. 5C) was observed and might activate other IG pathway genes indirectly.

In line with the protoplast data, ERF103 and ERF105 OE plants displayed a strong and significant increase in $C Y P 81 F 2$ expression, both in roots and in leaves. Activation of the IGbiosynthetic genes by ERF104 was less pronounced, presumably due to the low, but significant, overexpression level in roots (less than threefold). Although their overexpression was at an intermediate level, ERF107 and ERF106 activated $C Y P 81 F 2$, particularly in roots. Interestingly, MYB51 transcription (Fig. 5C) followed a pattern that was highly similar to that described for $C Y P 81 F 2$ (Fig. 5B).

IXc member ERF96 strongly induced $P D F 1.2 A$ in leaves in contrast to the group IXb ERF OE lines that showed limited activation of $P D F 1.2 A$. Moreover, this gene was not significantly upregulated in roots of several ERF OE plants. That indicates $P D F 1.2 A$ is not a strong candidate for mediating tolerance to $V$. longisporum in WT plant roots. These findings support distinct gene regulatory functions of ERF96 and group IXb ERF.

\section{Functional $C Y P 81 F 2$ is crucial in mounting defense against $V$. longisporum.}

Because the overexpression lines of ERF103 to ERF107 mediate resistance to $V$. longisporum and induce IG pathway genes, the impact of this pathway on defense against $V$. longisporum was evaluated. MYB51 and CYP81F2 were both strongly induced in $V$. longisporum-infected roots (Fig. 6A). Compared with WT plants, the cyp81f2 mutant showed no alteration in growth under mock treatment conditions (Fig. 6B), but when infected with $V$. longisporum, a significantly enhanced susceptibility was observed, as demonstrated by reduced fresh weight (Fig. 6C), increased disease symptoms (Fig. 6D), and elevated fungal DNA content in leaves (Fig. $6 \mathrm{E})$. However, increased susceptibility was less pronounced 
compared with the cyp79b2/b3 double mutant, which fully blocked Trp-derived secondary metabolism (Zhao et al. 2002).

\section{DISCUSSION}

Phenotypical AtTORF-Ex screening of infected stem sections enables rapid and reliable identification of TF determining fungal resistance.

Functional screens using transgenic gain-of-function plant collections were used, to identify both TF (Weiste et al. 2007) and non-TF genes (Ichikawa et al. 2006) involved in stress response or development. AtTORF-Ex screening has been proven to be successful in finding TF enhancing tolerance to various abiotic and biotic stresses (Catinot et al. 2015; Chen et al. 2016; Walper et al. 2016; Wehner et al. 2011b; Weiste et al. 2007). Here, a novel phenotypic screen based on colonization of the inflorescence stem, which is particularly suited for analyzing systemic spread of vascular pathogens, was applied. This assay was found to be simple and reliable, whereas attempts based on analyzing disease symptoms via projected

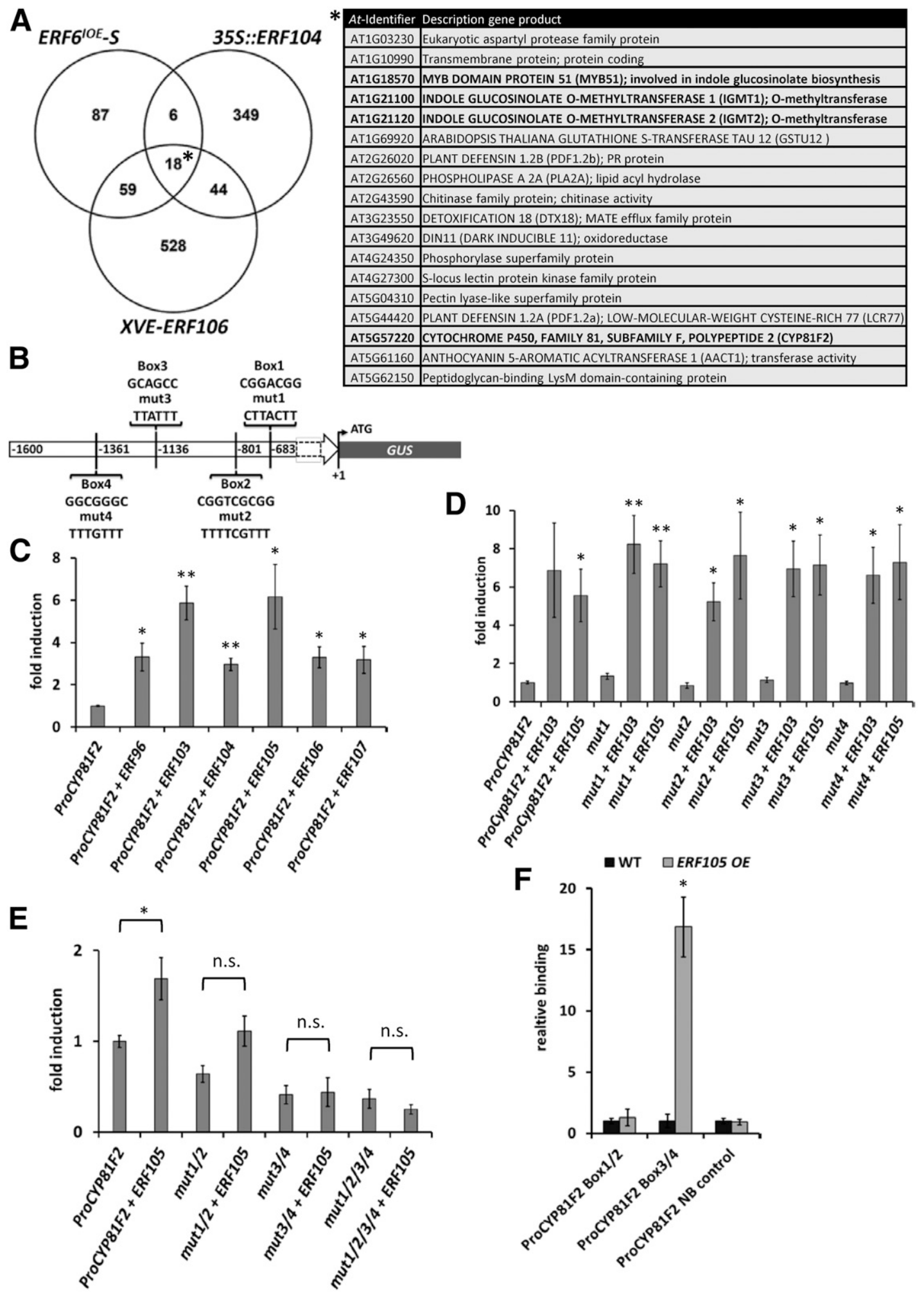


leaf area or fresh weight were less robust due to high variation of infected plant material. Alternatively, detection of fungal DNA by quantitative PCR (qPCR) is a highly reliable technique, but less suited for large screening approaches because it is labor- and cost-intensive. In general, molecular data derived by qPCR and phenotypical stem assays matched each other. However, the two group IXb members ERF102 (ERF5) and ERF103 (ERF6) were not recovered in the resistance screen, although ERF103 OE plants clearly had enhanced resistance, as was shown with qPCR. Based on statistical evaluation, the screen was saturated. Each ERF gene should be represented more than 14 times, resulting in $>99 \%$ coverage of all tested TF (Weiste et al. 2007). ERF102 and ERF103 overexpressors are represented in the AtTORF-Ex collection, as they were confirmed by sequencing. A possible explanation arises from the distinct experimental setups of both assays, as different tissues (rosette leaves versus stems) and sampling times (21 versus 30 dpi) were used. Moreover, overexpression might result in developmental alterations and growth differences, which may particularly influence the outcome of the stem assay.

\section{AtTORF-Ex screening is suited to identify candidate TF in large, potentially redundant gene families.}

The ERF TF family has been analyzed in a proof-of-principle experiment demonstrating that large $\mathrm{TF}$ gene families can easily be screened. The outcome, showing that most of the ERF family members (Licausi et al. 2013) did not lead to enhanced resistance to $V$. longisporum, except for a small subset of highly related group IX ERF, emphasizes the specificity of the approach. Although some group IX ERF have already been characterized as mediating defense-related gene expression (Huang et al. 2016), their impact on resistance to vascular pathogens has not yet been studied. In line with previous publications, single mutants of the examined ERF genes did not show altered susceptibility to several pathogens (Catinot et al. 2015; Huang et al. 2016; Xu et al. 2016). Therefore, (partial) functional redundancy can be assumed. Indeed, our findings highlight the advantages of this gain-of-function-approach, as it is particularly suited to identify groups of potentially redundant TF.

Although a canonical GCC-box element has been proposed as a binding site for most group IX ERF (Huang et al. 2016), overexpression of ERF96 and IXb members specifically restricted $V$. longisporum propagation. This may be due to differences in the in vivo DNA-binding specificity of ERF family members. Alternatively, properties other than DNA-binding are important for their function (e.g., adjacent DNA-binding motifs, interaction with other TF, or posttranslational modifications). ERF dimerization has been demonstrated (e.g., for ERF5), but its functional relevance is currently unresolved (Son et al. 2012). In this study, the CYP81F2 target promoter was activated by group IXb ERF using a protoplast reporter assay. Recently, a perfect GCCGCC element was identified 2.6-kb upstream the ATG start of $C Y P 81 F 2$ and was supported as an in vivo binding site by $\mathrm{ChIP}_{\mathrm{PCR}}$ experiments applying overexpressed ERF103 (Xu et al. 2016). Nevertheless, the exact binding site has not been mapped, nor has its in vivo impact on gene-expression been determined. Here, it was demonstrated that a 1.6-kb promoter fragment is sufficient for activation by all ERF analyzed in this study, although only GCC-like boxes (e.g., GCCNGCC, GCNGCC) were detected in the respective promoter fragment. Whereas single GCC-like box mutations had no impact on the reporter in protoplasts, particularly boxes 3 and 4 did functionally interact in ERF-mediated gene activation. This is in line with the $\mathrm{ChIP}_{\mathrm{PCR}}$ data evaluating direct promoter binding, as well as genome-wide DAPseq (DNA affinity purification sequencing) data demonstrating ERF binding in this area (O'Malley et al. 2016). Mutations in boxes 1 and 2 showed a minor effect and, therefore, may have limited impact. However, because no direct $\mathrm{ChIP}_{\mathrm{PCR}}$ signals were observed in this promoter region, group IXb ERF are proposed to regulate $C Y P 81 F 2$ via the redundantly acting boxes 3 and 4 .

Interestingly, one nongroup IX member of the ERF branch (ERF113/RAP2.6L; At5g13330) and one DREB factor (ERF60; At4g39780) have been found repeatedly in the AtTORF-Ex screen. Although not fully analyzed, ERF113 was found in a network-guided discovery of TF in aliphatic glucosinolate biosynthesis ( $\mathrm{Li}$ et al. 2018). In addition, ERF113 has been found to be involved in abscisic acid (ABA) and/or abiotic stress responses (Liu et al. 2012). Moreover, ERF60 has been implicated in operating in abiotic stress responses. Due to its complex life cycle, defenses against Verticillium spp. might be mounted at several phases of plant colonization, such as early root infection, during vascular transmission or propagation in leaves. It is well-known that induction of early senescence symptoms is crucial for efficient colonization and virulence of $V$. longisporum (Reusche et al. 2013). Instead of inducing classical defenses, ERF60 and ERF113 may control other mechanism to reduce fungal propagation (e.g., by altering overall plant physiology and development, senescence progression, or hormonal responses).

\section{Overexpression of ERF96 mediates enhanced resistance to $V$. longisporum but results in reduced growth.}

ERF96, which mediated resistance to V. longisporum, was by far the most frequently identified TF in the AtTORF-Ex screen (six times). It has been demonstrated that ERF96 acts as a transcriptional activator via its EDLL-activation domain (Catinot et al. 2015; Wang et al. 2015). Although ERF96 OE plants display enhanced resistance to necrotrophic pathogens such as the fungus Botrytis cinerea and the bacterium Pectobacterium carotovorum, they are more susceptible to salicylic acid (SA)dependent biotrophs such as Pseudomonas syringae (Catinot et al. 2015). Like the well-described ERF1 (ERF92) and ORA59 (ERF94) (Berrocal-Lobo et al. 2002; Pré et al. 2008; Zander et al. 2014), ERF96 is induced by JA and ET, binds

\footnotetext{
Fig. 4. $C Y P 81 F 2$ is a direct target of IXb ethylene response factor (ERF) transcription factors (TF). A, Venn diagram of transcriptome approaches comparing upregulated genes upon estradiol-induced expression of ERF106 (XVE-ERF106; gene expression with and without ERF106 induction was compared; $P \leq$ 0.001 ; fold change [FC] $\geq 2$ ); dexamethasone-induced expression of $E R F 103$ (=ERF $6_{-S}^{I O E}$ [Dubois et al. 2013]; gene expression with and without $E R F 103$ induction was compared; $P \leq 0.05$; FC $\geq 2$ ); or constitutive expression of ERF104 ( $335 S:: E R F 104$ [Bethke et al. 2009]; calculated with GEO accession GSE1 1807, relative to wild-type plants; $P \leq 0.05 ; \mathrm{FC} \geq 2$ ). A list of 18 commonly upregulated genes is provided on the right. B, Schematic view of the 1,600 -bp CYP81F2 promoter fused to the GUS reporter gene (ProCYP81F2:GUS). GCC-like boxes and the mutant versions used in parts C through F are labeled as Box 1 through 4 and mut 1 through 4 , respectively. Localization of cis-elements is provided relative to the ATG start (+1). C, Activation of the ProCYP81F2: GUS reporter by coexpression of the indicated ERF; $n=3$ biological replicates. D and E, Activation of single and multiple mutated versions of the ProCYP81F2 reporter by ERF103 and ERF105. GUS/NAN values ( \pm standard deviation) for $n=3$ biological replicates were calculated according to Ehlert et al. (2006). Fold induction values relative to transfection with only the respective reporter vector are shown. Student's $t$ test was used to compare values with ProCYP81F2:GUS without ERF; * indicates $P \leq 0.05$; ** indicates $P \leq 0.01$; n.s.: not significant (C through E). F, Chromatin immunoprecipitation coupled to PCR analysis of HA-tagged ERF105 binding to the CYP81F2 promoter (two biological and three technical replicates; the experiment was repeated twice with comparable results). Student's $t$ test; * indicates $P \leq 0.05$.
} 


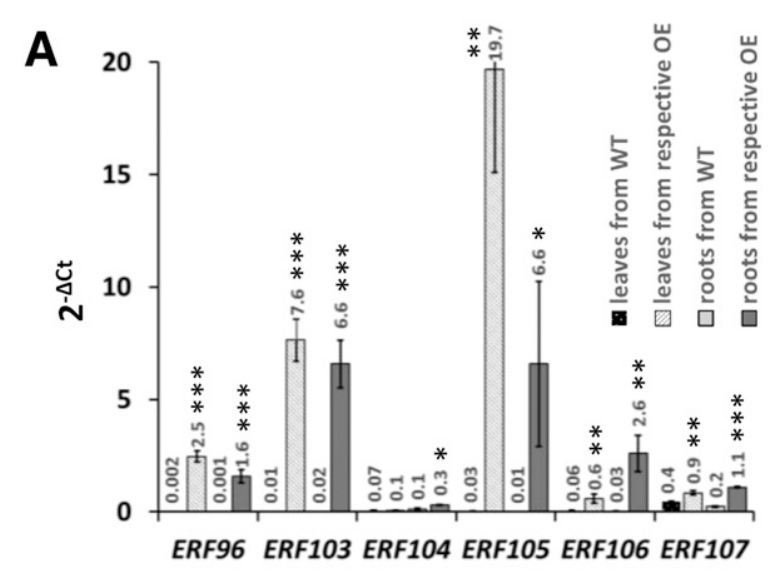

B
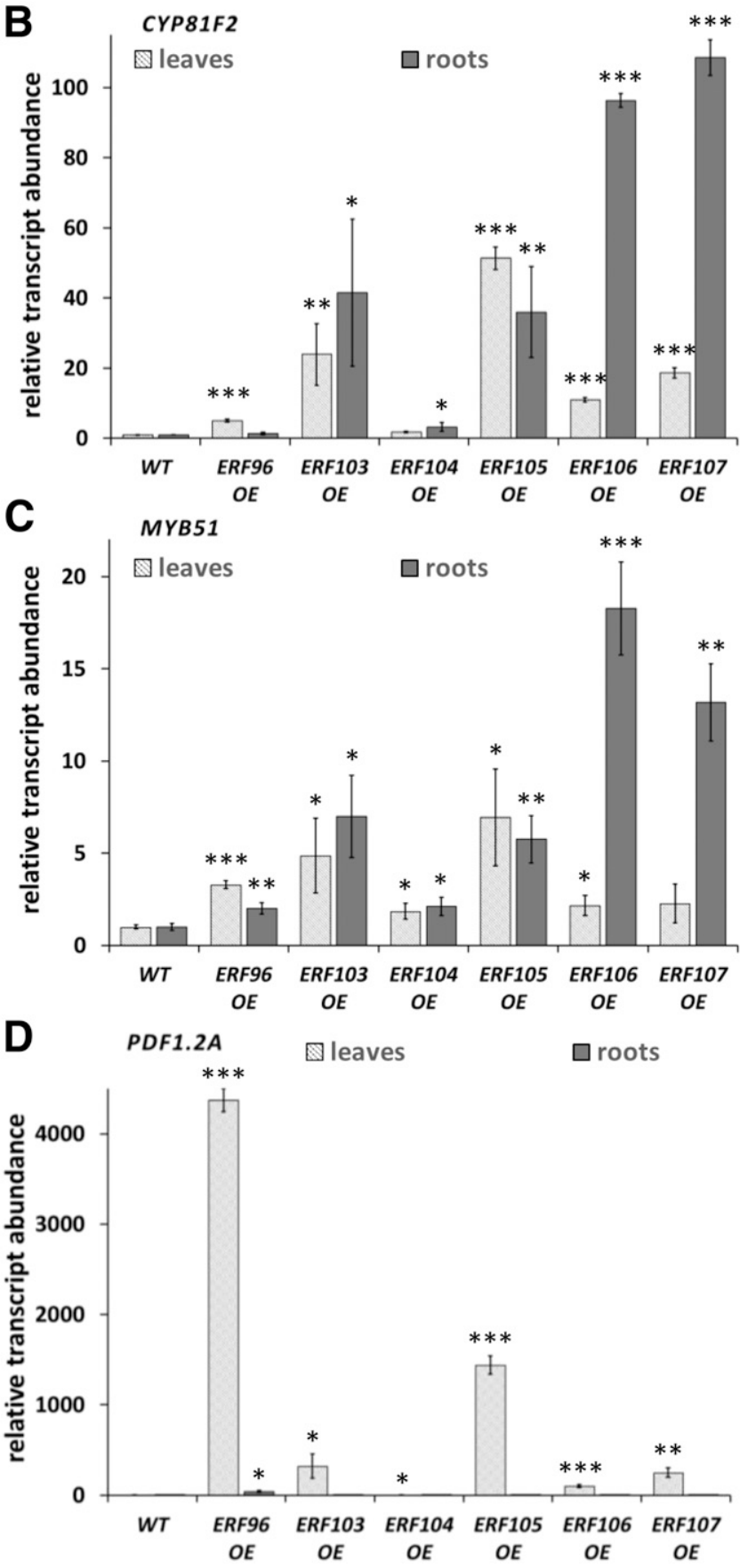

GCC boxes in vitro, and directly controls classical defense genes, such as $P D F 1.2, P R 3$, and PR4. In line with published transcriptome data (Catinot et al. 2015), our results confirmed activation of $P D F 1.2 A$ in ERF96 OE plants. Based on transcriptome studies of ERF96 OE plants and our transcriptional analyses upon $V$. longisporum infection, ERF96 differs considerably in its gene regulatory capacity from the other ERF studied.

Although ERF96 transcript abundance was increased in infected roots or late in leaves after the fungus reached the foliage, its expression level was very low. Consequently, it is unlikely that ERF96 plays an important role in mounting a defense against $V$. longisporum in WT plants.

ERF96 directly binds to and activates the closely related ORA59 promoter (Catinot et al. 2015), which has been identified as a master regulator of JA- and ET-controlled defenses (Chen et al. 2016; Pré et al. 2008), indicating a regulatory interplay among ERF1, ORA59, and ERF96 in JA and ET signaling. Whereas JA biosynthesis and signaling mutants do not effect susceptibility to $V$. longisporum (Johansson et al. 2006; Ralhan et al. 2012), mutant analyses and feeding experiments with ET precursors support the impact of ET in resistance against $V$. longisporum (Depotter et al. 2016). Nevertheless, aside from ERF96, neither ERF1 nor ORA59 were picked up in the screen, indicating that resistance to Verticillium spp. depends on a still unknown mechanism. Recently, ERF96 OE plants were shown to be hypersensitive to ABA and effect growth under water-limiting conditions and water-use efficiency (Dubois et al. 2013; Wang et al. 2015). Partial impairment in growth under nonstressed conditions was confirmed in this study. Due to the complex infection and colonization pathway, $V$. longisporum propagation depends on plant physiology and development. Hence, ERF96 OE may impact resistance toward $V$. longisporum through its effect on the overall plant performance under stress.

Because single ERF96 RNAi lines have not been found to enhance susceptibility toward $V$. longisporum, we assumed functional redundancy between closely related ERF (ERF95, ERF96, ERF97). However, the triple loss-of-function approach showed fungal propagation comparable to WT plants, which is in line with the very low expression of ERF95, ERF96, and ERF97. Moreover, no ERF97 and only one ERF95 OE line was recovered from the AtTORF-Ex screen, supporting a specific function of ERF96 in mediating resistance to V. longisporum, which is not shared by the other subgroup IXc members.

\section{The highly related subgroup IXb ERF} counteract $V$. longisporum infection in a redundant manner.

In addition to ERF96, four highly related IXb members (ERF104, ERF105, ERF106, ERF107) were identified that showed enhanced resistance in the AtTORF-Ex screen, as well as reduced amounts of fungal DNA. So far, only ERF104 has

Fig. 5. Activation of ethylene response factor (ERF)-regulated pathogen defense genes in noninfected $E R F$-overexpressing (OE) plants. A, Transcript abundance in leaves and roots of 14-day-old wild-type (WT) and the respective ERF OE plants grown on solid MS medium. Values were determined by quantitative reverse transcription PCR (qRT-PCR) analysis. $2^{-\Delta \mathrm{Ct}}$ values were normalized to UBQ5. Mean values ( \pm standard deviation) are shown for $n=3$ biological replicates. Student's $t$ test was used to compare $E R F$ OE values from leaves or roots with respective WT plant values; * indicates $P \leq 0.05$; ** indicates $P \leq 0.01$; *** indicates $P \leq 0.001$. Relative transcript abundance of $\mathbf{B}, C Y P 81 F 2, \mathbf{C}, M Y B 51$, and $\mathbf{D}, P D F 1.2 A$ in the $E R F$ OE plants analyzed in part A. Results for leaves (light gray) and roots (dark gray) are provided separately. For parts B through $\mathrm{D}$, mean values ( \pm standard deviation) for $n=3$ biological replicates relative to WT plants (set to 1) are shown. Student's $t$ test was used to compare values with WT plants; * indicates $P \leq 0.05$; ** indicates $P \leq 0.01$; *** indicates $P \leq 0.001$. 
been functionally characterized as participating in ETdependent basal resistance (Bethke et al. 2009). Importantly, ERF104 is known to be controlled posttranslationally by phosphorylation via MPK3/6, which are important players in pathogen signaling. A similar regulation has also been demonstrated for the closely related group IXb members ERF102 (AtERF5) and ERF103 (AtERF6) (Meng et al. 2013; Xu et al. 2016), but the regulatory mechanism-how MPK3/6 phosphorylation controls ERF function-remains to be elucidated. Importantly, ERF102 to ERF105 share conserved MPK phosphorylation sites, indicating a similar posttranslational regulation, whereas ERF106 and ERF107 lack these sites due to a C-terminal truncation. Consequently, these data suggest common regulation, at least between ERF102, ERF103, ERF104, and ERF105. Contradictory findings have been published on the function of ERF102 and ERF103 in JA and ET- and SA-mediated defenses (e.g., that the erf102/erf103 [erf5erf6] double mutant was found to be more susceptible to $B$. cinerea [Moffat et al. 2012] or showed a WT response [Meng et al. 2013]). In our study, the erf102/erf103 double mutant was more susceptible to $V$. longisporum.

(Partial) redundancy between ERF102 to ERF107 can be assumed from the gain-of-function-screen and because none of the other single and double loss-of-function lines showed increased susceptibility. The close genomic vicinity of several $E R F$ genes hampered generation of higher order mutants. Gene-editing techniques may reveal insights into the redundancy of the subgroup IXb ERF.

With respect to their transcriptional regulation in response to V. longisporum, the subgroup IXb ERF differ considerably. The
$V$. longisporum infection process is complex, and defenses may be mounted, for example, during early root infection, translocation to the vasculature, transport in the xylem, or release into the leaf. Hence, we studied $E R F$ gene induction that was dependent on tissue and infection time. Whereas ERF102, ERF103, and ERF105 were similarly induced in roots, ERF104 and $E R F 107$ already showed high background expression in the uninduced state. In addition, we recovered only low-level overexpressors of ERF104 and ERF107, showing 2.5- or 4.7fold transcript levels in roots compared with WT plants. Because ERF104 is regulated on a posttranslational level, transcriptional activation by pathogens is not necessarily required. It is interesting to note, that ERF104 and ERF107 are arranged on the genomic DNA in tandem and presumably have arisen through gene duplication. Although ERF107 is lacking in the conserved MPK phosphorylation sites, alternative regulation by phosphorylation cannot be excluded.

Recent publications have proposed that ERF106 (DEWAX2) and ERF107 (DEWAX) function as negative regulators of cuticle development, although ERF107 was demonstrated to be a transcriptional activator (Go et al. 2014; Kim et al. 2018). Consequently, ERF107 OE plants display increased resistance to the necrotrophic leaf pathogen B. cinerea (Ju et al. 2017). Our transcriptome studies, which applied an inducible expression of ERF106, did not reveal differentially expressed genes related to cuticle development. Differences may be explained by focusing on primary targets due to short-term induction of the TF or plant age. Nevertheless, cuticle development should not influence root vascular pathogens like V. longisporum.
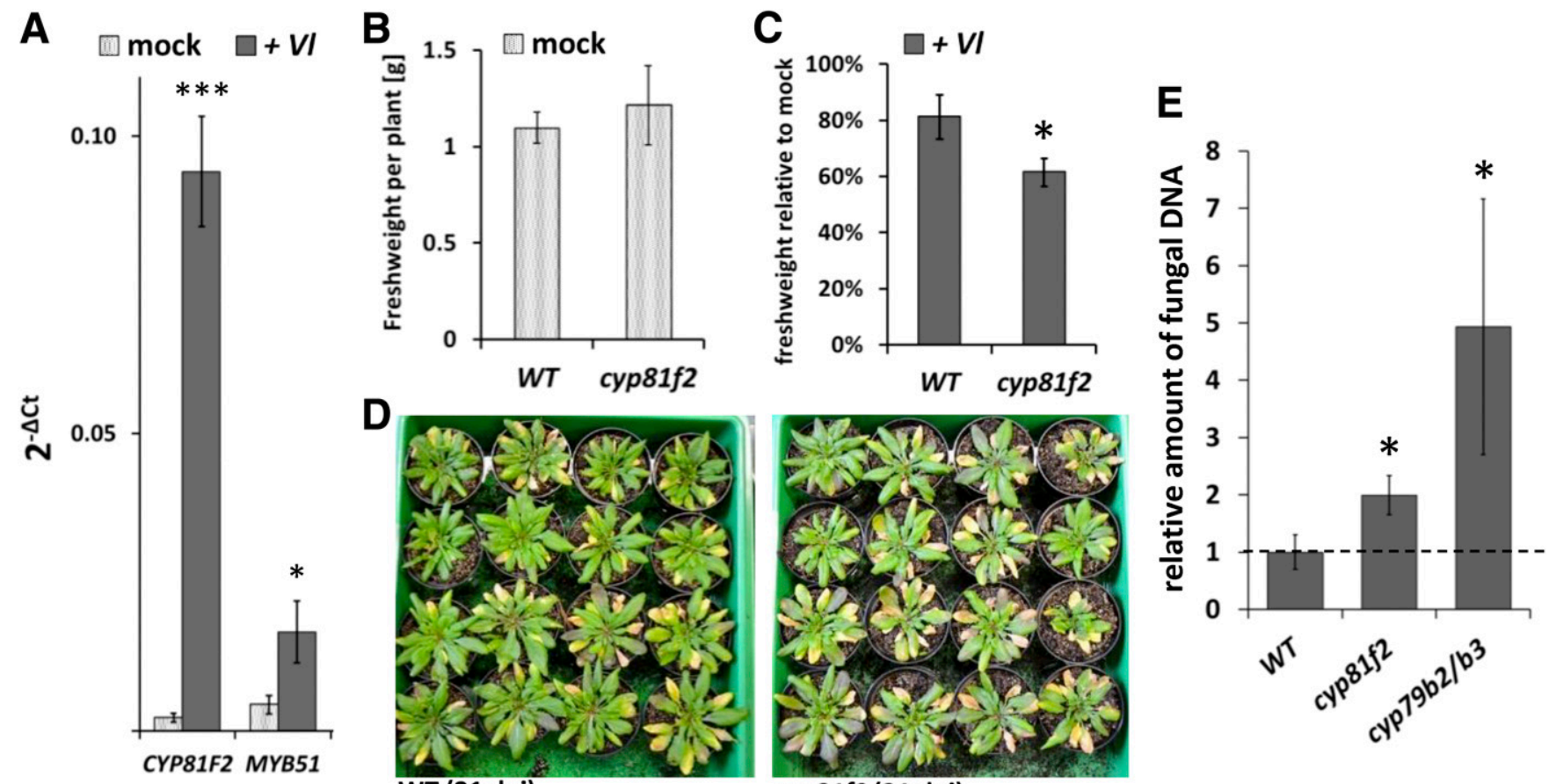

Fig. 6. Antimicrobial indole glucosinolates are crucial for mounting a defense to Verticillium longisporum, as determined by analyzing the cyp81f2 mutant. A, Transcriptional activation of CYP81F2 and MYB51 in Arabidopsis thaliana roots infected with V. longisporum, as determined by quantitative reverse transcription PCR (qRT-PCR) at 6 days postinoculation (dpi). Mean values ( \pm standard deviation) are shown for $n=3$ biological replicates. $2^{-\Delta C t}$ values were normalized to $U B Q 5$. Student's $t$ test was used to compare values with mock-treated plants; * indicates $P \leq 0.05$; *** indicates $P \leq 0.001$. B, Mock-treated and soil-grown 6-week-old wild-type (WT) and cyp81f2 plants had comparable growth, as demonstrated by quantifying fresh weight per plant; $n=5$ plants each. $\mathbf{C}$, Quantification of $V$. longisporum-caused reduction of fresh weight at 21 dpi. The relative fresh weight represents the ratio of $V$. longisporum-inoculated to mock-treated plants (mock $=100 \%$ ). Mean values ( \pm standard deviation) were obtained for $n=3$ biological replicates consisting of rosette leaves from five pooled plants. Student's $t$ test was used to compare values with WT plants; * indicates $P \leq 0.05$. D, Representative pictures showing rosette leaves of infected WT and cyp81f2 mutant plants at $21 \mathrm{dpi}$. E, Resistance assay analyzing fungal DNA in leaf rosettes at $21 \mathrm{dpi}$. Mean values ( \pm standard deviation) of fungal DNA in cyp81f2 and cyp 79b2/b3 mutants are shown relative to the infected WT plants (dotted line); $n=3$ biological replicates consisting of the rosette leaves from five pooled plants. Student's $t$ test was used to compare values with WT plants; * indicates $P \leq 0.05$. 
Indeed, GENEVESTIGATOR analysis of multiple stress treatments, expression analyses after $V$. longisporum infection, and impairment in growth support a distinct functionality of ERF106 and ERF107 compared with the other IXb ERF. Although subgroup IXb ERF share related properties (such as impact on IG metabolism), they also show distinct features and transcriptional or posttranscriptional regulations. Based on the expression data, some ERF might be crucial in mounting especially early defenses in roots. Surprisingly, transcription of all subgroup IXb ERF genes was significantly downregulated in leaves upon infection, particularly at late time points (21 dpi). This finding might be explained by a pathogenic virulence factor, which is suppressing important ERF-mediated plant defenses.

\section{Subgroup IXb ERF control pathogen-induced IG biosynthesis to counteract $V$. longisporum infection.}

To define ERF-dependent gene regulation and resistance mechanisms toward $V$. longisporum, public ERF overexpression data sets were mined and transcriptome studies on inducible expression of ERF106 were performed, because currently no transcriptome data are available for this TF. It should be noted that plant growth conditions, experimental procedures, and bioinformatic analyses differ considerably among these studies. Hence, these comparisons should be evaluated with care. Classical $P R$ genes, as well as genes involved in Trp-derived secondary metabolism, were detected as potential targets. In contrast to ERF96, overexpression of all group IXb TF displayed an enrichment of genes involved in the biosynthesis of Trp-derived antimicrobial compounds. Therefore, we focused on regulation of $M Y B 51$ and $C Y P 81 F 2$, which encode for a transcriptional master regulator of the IG pathway and a pathogen-induced IG biosynthetic gene, respectively. All group IXb ERF significantly activated the $C Y P 81 F 2$ promoter in protoplasts. Moreover, transgenic ERF OE plants redundantly activated $C Y P 81 F 2$ and MYB51 depending on the overexpression levels of the particular ERF. Interestingly, activation of $M Y B 51$ and $C Y P 81 F 2$ followed a very similar induction. It remains to be investigated whether this was due to a feedback of the activated IG pathway on its master regulator gene, $M Y B 51$, or whether group IXb ERF directly target the $M Y B 51$ promoter. More unanswered questions regarding the gene regulation of the IG pathway need to be addresses (e.g., the binding of MYB51 to promoters of IG metabolic genes has not been confirmed by $\left.\mathrm{ChIP}_{\mathrm{PCR}}\right)$.

Moreover, mutant analysis of cyp $81 f 2$ plants displayed significantly increased susceptibility, supporting the view that the IG pathway is a strong path for mounting resistance to $V$. longisporum. This is in line with previous findings, in which cyp81f2 mutants showed a tendency toward increased fungal DNA (Iven et al. 2012). Nevertheless, the $c y b 79 b 2 / b 3$ double mutant, which fully blocks the flux to Trp-derived secondary metabolism, was even more susceptible, suggesting other Trp-derived antimicrobial compounds may work additively, as previously described for $V$. longisporum and other pathogens (Iven et al. 2012; Sanchez-Vallet et al. 2010).

These findings suggest this is a stringent and reproducible screening procedure, which now can be used for other TF families available in the AtTORF-Ex-collection (Wehner et al. $2011 \mathrm{~b}$ ) to identify novel TF mediating resistance to specific pathogens, especially vascular fungi. Based on our results, ERF103 to ERF105 are the most promising candidates for crop improvement strategies, as their overexpression did not considerably influence plant growth. Advanced breeding and geneediting techniques provide further opportunities to enhance gene expression in a more defined manner than using classical overexpression approaches. This may circumvent reductions in growth, as was observed here with overexpression of ERF96, $E R F 106$, and $E R F 107$. Based on these findings, the ERF genes examined in this study are valuable candidates for crop improvement strategies to generate $V$. longisporum-resistant oilseed rape.

\section{MATERIALS AND METHODS}

\section{Plant culture, fungal strains, infection procedures, and susceptibility assays.}

A. thaliana ecotype Col-0 was used throughout this study. Details on transgenic lines are provided in Supplementary Table S4. All seeds were surface-sterilized by incubation with chlorine gas for $3 \mathrm{~h}$. To synchronize germination, seeds were stratified at $4^{\circ} \mathrm{C}$ for 3 days.

For infection experiments, V. longisporum V143 (Zeise and von Tiedemann 2002) or a gLUC-V143 strain (J. Utermark and P. Karlovsky, University of Göttingen) was used. Cultivation and harvesting of $V$. longisporum spores were performed as described in Iven et al. (2012). Infection was performed with freshly harvested conidiospores.

Screening of the AtTORF-Ex collection (Weiste et al. 2007) was performed by growing plants in a $1: 1$ ( $\mathrm{vol} / \mathrm{vol})$ soil/sand mixture for 16 days $\left(16 \mathrm{~h}\right.$ of light and $8 \mathrm{~h}$ of dark; $22^{\circ} \mathrm{C} ; 60 \%$ relative humidity $[\mathrm{RH}])$. After excavating the plants, roots were washed and inoculated by dipping in a petri dish holding $25 \mathrm{ml}$ of $V$. longisporum spore solution $\left(1 \times 10^{6}\right.$ spores $\left./ \mathrm{ml}\right)$ for $40 \mathrm{~min}$ before repotting the plants in regular soil. After 30 days, 1 to $1.5 \mathrm{~cm}$ stem segments were cut, surface-sterilized $(5 \mathrm{~min}$ in $70 \%$ ethanol; 5 min in $0.02 \%$ sodium hydrogen chloride solution supplemented with $0.02 \%$ Triton X-100), and thoroughly washed with sterile water. Finally, the stem segments were placed on potato dextrose broth agar (Sigma-Aldrich Chemie $\mathrm{GmbH}$ ), supplemented with $500 \mathrm{mg}$ of cefotaxim per liter (Duchefa). After incubation in darkness for 3 to 5 days at room temperature, fungal mycelia became visible.

To determine fungal DNA in A. thaliana rosette leaves using qPCR, the infection procedure was further optimized. Plants were cultivated for 21 days under long-day conditions in a 3:1 (v/v) soil/sand mixture (bird sand from Pet Bistro) in pots $(7 \mathrm{~cm})$. To reduce variability, plants of similar size were carefully selected before starting the experiment. For root-dip inoculation, the roots were washed and dipped for $60 \mathrm{~min}$ in a petri dish holding $25 \mathrm{ml}$ of a spore suspension $\left(2 \times 10^{6}\right.$ spores/ml) in $1 / 4 \mathrm{MS}$ liquid medium.

For in vitro root infection assays, a petri dish-based cultivation system on agarose-solidified Murashige and Skoog medium (1.5 g/l) was applied as described in Iven et al. (2012). Seedlings were grown for 11 days under long-day conditions, and roots were infected with $4 \times 10^{5}$ spores $/ \mathrm{ml}$. Roots or rosettes were harvested for qRT-PCR analyses at 3 or 6 dpi.

Infection with Pseudomonas syringae pv. tomato DC3000 was performed (Jacob et al. 2017) to characterize the erf95/erf97amiRerf96 mutant line.

Projected leaf area was determined using BlattFlaeche software (Datinf $\mathrm{GmbH}$ ) as described in Iven et al. (2012).

The fresh weight of $A$. thaliana rosettes was quantified, after removing roots and stems, by measuring the weight of five pooled rosettes. Each experiment was carried out at least three times with five pooled plants each.

\section{Vector construction and plant transformation.}

In order to generate the ProCYP81F2:GUS reporter construct, the 1600 bp $C Y P 81 F 2$ promotor region, including the 5' UTR was PCR-amplified using promotor-specific primers 
(Supplementary Table S3), which introduce flanking NcoI and BamHI restriction sites to the PCR-fragment. By using the respective restriction enzymes, the promotor fragment was inserted upstream the GUS-reporter gene into the pBT10:GUS vector (Bernd Weisshaar; University of Bielefeld). To mutate putative ERF-binding sites (GCC-like boxes), the QuikChange II Site-Directed Mutagenesis Kit (Agilent Technologies Inc., USA) was applied using primers.

amiR constructs were designed to silence ERF96 or both ERF 104 and ERF105 by applying the WMD3 protocol and the plasmid pRS300 as template (Schwab et al. 2006). Via GATEWAY recombination sites attached to the primers, the PCR-amplified fragment was recombined in the entry vector pDONR201 (Invitrogen) by BP reaction. In a subsequent LR reaction, the binary GATEWAY-compatible vector pB2GW7 was used, which enabled expression of the amiR construct under control of the $35 \mathrm{~S}$ promoter (C. Thurow, University of Göttingen). Plant transformation procedures were performed according to Weigel and Glazebrook (2002) using Agrobacterium tumefaciens GV3101.

\section{Molecular biology methods.}

PCR analyses for genotyping the selected AtTORF-Ex plant lines was performed using primers as previously described by Weiste et al. (2007).

Fungal propagation in planta was determined by qPCR analyzing fungal DNA (Iven et al. 2012). The plant material was ground in liquid nitrogen, and $100 \mathrm{mg}$ of powder was used to extract DNA. PCR analysis was performed using $100 \mathrm{ng}$ of DNA and the $V$. longisporum-specific primers OLG70 and OLG71 (Eynck et al. 2007). Fungal DNA content was calculated relative to an ACTIN8 amplicon derived from Arabidopsis genomic DNA. Each biological replicate was obtained by pooling rosettes from five infected plants. At least three replicates were used to calculate one fungal DNA data point.

For qRT-PCR studies, each RNA sample was isolated from roots or leaves following a TRIzol protocol (Chomczynski and Mackey 1995). After $30 \mathrm{~min}$ of DNase I treatment (Thermo Fisher Scientific), cDNA was synthesized from $1 \mu \mathrm{g}$ of total RNA using oligo(dT) primers, random nonamer primers, and the reverse transcription RevertAid H Minus (Thermo Fisher Scientific) according to the manufacturer's manual. Amplification products were visualized by SYBR green. The following conditions were generally applied: $10 \mathrm{~min}$ at $95^{\circ} \mathrm{C}, 40$ cycles of $20 \mathrm{~s}$ at $95^{\circ} \mathrm{C}, 20 \mathrm{~s}$ at $56^{\circ} \mathrm{C}$, and $20 \mathrm{~s}$ at $72^{\circ} \mathrm{C}$, followed by a default dissociation stage program to detect nonspecific amplification. Values were calculated from at least three biological replicates, either using the $2^{-\Delta \mathrm{CT}}$ method or as fold induction values using the $2^{-\Delta \Delta C T}$ method (Livak and Schmittgen 2001), using $U B Q 5$ expression levels for normalization. $\mathrm{ChIP}_{\mathrm{PCR}}$ was performed using primers, as previously described in Weiste and Dröge-Laser (2014).

\section{RNA sequencing.}

Generation of XVE-ERF106 plants was as described in Walper et al. (2016). Seedlings (14-day-old) were grown on solidified MS media and induced with $5 \mathrm{ml}$ of an Est solution (10 $\mu \mathrm{M}$ in water) compared with the control group without Est. RNA extraction, library preparation, and sequencing were as described in Pedrotti et al. (2018).

\section{Protoplast transfection assays.}

Mesophyll protoplasts were isolated from rosette leaves of 4-week-old $A$. thaliana WT plants grown on soil (12 h of light/12 h of dark; $22^{\circ} \mathrm{C} ; 60 \% \mathrm{RH}$ ) following the procedure described by Weiste and Dröge-Laser (2014). Generation of effector plasmids was as described in Wehner et al. (2011a). Plasmid DNA was amplified in the Escherichia coli DH5 $\alpha$ and prepared using a Qiagen plasmid midi kit (Qiagen). GUS/NAN ratios were measured according to Ehlert et al. (2006). Calculations were based on three biological replicates.

\section{Statistics and software.}

Statistical tests were performed with Microsoft Excel software. XVE-ERF106 RNAseq data were analyzed with the publicly accessible $\mathrm{R}$ studio edgeR package. Open access transcriptome data and XVE-ERF106 data were compared in Venn diagrams using the online tool Venny. Protein sequence alignments were performed with the online tool from the MAFFT (multiple alignment using fast fourier transform) server at EBI.

\section{ACKNOWLEDGMENTS}

We thank P. Karlovsky and C. Thurow (University of Göttingen) for providing an unpublished LUC-tagged Verticillium longisporum strain and vector constructs, respectively. We thank J. Heeg and A. Marsell (University of Würzburg) for their excellent assistance.

\section{AUTHOR-RECOMMENDED INTERNET RESOURCES}

GENEVESTIGATOR database: https://genevestigator.com/gv

MAFFT server: https://www.ebi.ac.uk/Tools/msa/mafft

WMD3 protocol: http://wmd3.weigelworld.org

Venny online Venn diagram tool: http://bioinfogp.cnb.csic.es/tools/venny/index.html

\section{LITERATURE CITED}

Ahuja, I., Kissen, R., and Bones, A. M. 2012. Phytoalexins in defense against pathogens. Trends Plant Sci. 17:73-90.

Allen, M. D., Yamasaki, K., Ohme-Takagi, M., Tateno, M., and Suzuki, M. 1998. A novel mode of DNA recognition by a beta-sheet revealed by the solution structure of the GCC-box binding domain in complex with DNA. EMBO J. 17:5484-5496.

Bednarek, P., Pislewska-Bednarek, M., Svatos, A., Schneider, B., Doubsky, J., Mansurova, M., Humphry, M., Consonni, C., Panstruga, R., SanchezVallet, A., Molina, A., and Schulze-Lefert, P. 2009. A glucosinolate metabolism pathway in living plant cells mediates broad-spectrum antifungal defense. Science 323:101-106.

Berrocal-Lobo, M., and Molina, A. 2008. Arabidopsis defense response against Fusarium oxysporum. Trends Plant Sci. 13:145-150.

Berrocal-Lobo, M., Molina, A., and Solano, R. 2002. Constitutive expression of ETHYLENE-RESPONSE-FACTOR1 in Arabidopsis confers resistance to several necrotrophic fungi. Plant J. 29:23-32.

Bethke, G., Unthan, T., Uhrig, J. F., Pöschl, Y., Gust, A. A., Scheel, D., and Lee, J. 2009. Flg22 regulates the release of an ethylene response factor substrate from MAP kinase 6 in Arabidopsis thaliana via ethylene signaling. Proc. Natl. Acad. Sci. U.S.A. 106:8067-8072.

Catinot, J., Huang, J.-B., Huang, P.-Y., Tseng, M.-Y., Chen, Y. L., Gu, S.-Y., Lo, W.-S., Wang, L.-C., Chen, Y.-R. Y.-L., and Zimmerli, L. 2015. ETHYLENE RESPONSE FACTOR 96 positively regulates Arabidopsis resistance to necrotrophic pathogens by direct binding to GCC elements of jasmonate - and ethylene-responsive defence genes. Plant Cell Environ. 38:2721-2734.

Chen, H.-Y., Hsieh, E.-J., Cheng, M.-C., Chen, C.-Y., Hwang, S.-Y., and Lin, T.-P. 2016. ORA47 (octadecanoid-responsive AP2/ERF-domain transcription factor 47) regulates jasmonic acid and abscisic acid biosynthesis and signaling through binding to a novel cis-element. New Phytol. 211:599-613.

Chomczynski, P., and Mackey, K. 1995. Short technical reports. Modification of the TRI reagent procedure for isolation of RNA from polysaccharide- and proteoglycan-rich sources. Biotechniques 19: 942-945.

Clay, N. K., Adio, A. M., Denoux, C., Jander, G., and Ausubel, F. M. 2009. Glucosinolate metabolites required for an Arabidopsis innate immune response. Science 323:95-101.

Depotter, J. R. L., Deketelaere, S., Inderbitzin, P., Tiedemann, A. V., Höfte, M., Subbarao, K. V., Wood, T. A., and Thomma, B. P. H. J. 2016. Verticillium longisporum, the invisible threat to oilseed rape and other brassicaceous plant hosts. Mol. Plant Pathol. 17:1004-1016. 
Dubois, M., Skirycz, A., Claeys, H., Maleux, K., Dhondt, S., De Bodt, S., Vanden Bossche, R., De Milde, L., Yoshizumi, T., Matsui, M., and Inzé, D. 2013. ETHYLENE RESPONSE FACTOR6 acts as a central regulator of leaf growth under water-limiting conditions in Arabidopsis. Plant Physiol. 162:319-332.

Ehlert, A., Weltmeier, F., Wang, X., Mayer, C. S., Smeekens, S., VicenteCarbajosa, J., and Dröge-Laser, W. 2006. Two-hybrid protein-protein interaction analysis in Arabidopsis protoplasts: Establishment of a heterodimerization map of group $\mathrm{C}$ and group $\mathrm{S}$ bZIP transcription factors. Plant J. 46:890-900.

Eynck, C., Koopmann, B., Grunewaldt-Stoecker, G., Karlowsky, P., and von Tiedemann, A. 2007. Differential interactions of Verticillium longisporum and $V$. dahliae with Brassica napus with molecular and histological techniques. Eur. J. Plant Pathol. 118:259-274.

Frerigmann, H., and Gigolashvili, T. 2014. MYB34, MYB51, and MYB122 distinctly regulate indolic glucosinolate biosynthesis in Arabidopsis thaliana. Mol. Plant 7:814-828.

Gigolashvili, T., Berger, B., Mock, H. P., Müller, C., Weisshaar, B., and Flügge, U. I. 2007. The transcription factor HIG1/MYB51 regulates indolic glucosinolate biosynthesis in Arabidopsis thaliana. Plant J. 50: 886-901.

Go, Y. S., Kim, H., Kim, H. J., and Suh, M. C. 2014. Arabidopsis cuticular wax biosynthesis is negatively regulated by the DEWAX gene encoding an AP2/ERF-type transcription factor. Plant Cell 26:1666-1680.

Guo, A., He, K., Liu, D., Bai, S., Gu, X., Wei, L., and Luo, J. 2005. DATF: A database of Arabidopsis transcription factors. Bioinformatics 21: 2568-2569.

Huang, P.-Y., Catinot, J., and Zimmerli, L. 2016. Ethylene response factors in Arabidopsis immunity. J. Exp. Bot. 67:1231-1241.

Ichikawa, T., Nakazawa, M., Kawashima, M., Iizumi, H., Kuroda, H., Kondou, Y., Tsuhara, Y., Suzuki, K., Ishikawa, A., Seki, M., Fujita, M., Motohashi, R., Nagata, N., Takagi, T., Shinozaki, K., and Matsui, M. 2006. The FOX hunting system: An alternative gain-of-function gene hunting technique. Plant J. 48:974-985.

Iven, T., König, S., Singh, S., Braus-Stromeyer, S. A., Bischoff, M., Tietze, L. F., Braus, G. H., Lipka, V., Feussner, I., and Dröge-Laser, W. 2012. Transcriptional activation and production of tryptophan-derived secondary metabolites in Arabidopsis roots contributes to the defense against the fungal vascular pathogen Verticillium longisporum. Mol. Plant 5: 1389-1402.

Jacob, C., Panchal, S., and Melotto, M. 2017. Surface inoculation and quantification of Pseudomonas syringae population in the Arabidopsis leaf apoplast. Bio Protoc. 7:3047-3054.

Johansson, A., Staal, J., and Dixelius, C. 2006. Early responses in the Arabidopsis-Verticillium longisporum pathosystem are dependent on NDR1, JA- and ET-associated signals via cytosolic NPR1 and RFO1. Mol. Plant-Microbe Interact 19:958-969.

Ju, S., Go, Y. S., Choi, H. J., Park, J. M., and Suh, M. C. 2017. DEWAX transcription factor is involved in resistance to Botrytis cinerea in Arabidopsis thaliana and Camelina sativa. Front. Plant Sci. 8:1210.

Kim, H., Go, Y. S., and Suh, M. C. 2018. DEWAX2 transcription factor negatively regulates cuticular wax biosynthesis in Arabidopsis leaves. Plant Cell Physiol. 59:966-977.

Li, B., Tang, M., Nelson, A., Caligagan, H., Zhou, X., Clark-Wiest, C., Ngo, R., Brady, S. M., and Kliebenstein, D. J. 2018. Network-guided discovery of extensive epistasis between transcription factors involved in aliphatic glucosinolate biosynthesis. Plant Cell 30:178-195.

Licausi, F., Ohme-Takagi, M., and Perata, P. 2013. APETALA2/ethylene responsive factor $(\mathrm{AP} 2 / \mathrm{ERF})$ transcription factors: Mediators of stress responses and developmental programs. New Phytol. 199:639-649.

Liu, P., Sun, F., Gao, R., and Dong, H. 2012. RAP2.6L overexpression delays waterlogging induced premature senescence by increasing stomatal closure more than antioxidant enzyme activity. Plant Mol. Biol. 79:609-622.

Livak, K. J., and Schmittgen, T. D. 2001. Analysis of relative gene expression data using real-time quantitative PCR and the $2(-\triangle \Delta C \mathrm{~T})$ Method. Methods 25:402-408.

Meng, X., Xu, J., He, Y., Yang, K.-Y., Mordorski, B., Liu, Y., and Zhang, S. 2013. Phosphorylation of an ERF transcription factor by Arabidopsis MPK3/MPK6 regulates plant defense gene induction and fungal resistance. Plant Cell 25:1126-1142.

Moffat, C. S., Ingle, R. A., Wathugala, D. L., Saunders, N. J., Knight, H., and Knight, M. R. 2012. ERF5 and ERF6 play redundant roles as positive regulators of JA/Et-mediated defense against Botrytis cinerea in Arabidopsis. PLoS One 7:e35995.

Nakano, T., Suzuki, K., Fujimura, T., and Shinshi, H. 2006. Genome-wide analysis of the ERF gene family in Arabidopsis and rice. Plant Physiol. 140:411-432.
Ohme-Takagi, M., and Shinshi, H. 1995. Ethylene-inducible DNA binding proteins that interact with an ethylene-responsive element. Plant Cell 7: 173-182.

O’Malley, R. C., Huang, S. C., Song, L., Lewsey, M. G., Bartlett, A., Nery, J. R., Galli, M., Gallavotti, A., and Ecker, J. R. 2016. Cistrome and epicistrome features shape the regulatory DNA landscape. Cell 165: 1280-1292.

Parisi, K., Shafee, T. M. A., Quimbar, P., van der Weerden, N. L., Bleackley, M. R., and Anderson, M. A. 2019. The evolution, function and mechanisms of action for plant defensins. Semin. Cell Dev. Biol. 88: 107-118.

Pedrotti, L., Weiste, C., Nägele, T., Wolf, E., Lorenzin, F., Dietrich, K., Mair, A., Weckwerth, W., Teige, M., Baena-González, E., and DrögeLaser, W. 2018. Snf1-RELATED KINASE1-controlled C/S -bZIP $^{\text {-b }}$ signaling activates alternative mitochondrial metabolic pathways to ensure plant survival in extended darkness. Plant Cell 30:495-509.

Pérez-Rodríguez, P., Riaño-Pachón, D. M., Guedes Corrêa, L. G., Rensing, S. A., Kersten, B., and Mueller-Roeber, B. 2010. PlnTFDB: Updated content and new features of the plant transcription factor database. Nucleic Acids Res. 38 (suppl_1):D822-D827.

Pfalz, M., Mikkelsen, M. D., Bednarek, P., Olsen, C. E., Halkier, B. A., and Kroymann, J. 2011. Metabolic engineering in Nicotiana benthamiana reveals key enzyme functions in Arabidopsis indole glucosinolate modification. Plant Cell 23:716-729.

Pfalz, M., Vogel, H., and Kroymann, J. 2009. The gene controlling the Indole Glucosinolate Modifierl quantitative trait locus alters indole glucosinolate structures and aphid resistance in Arabidopsis. Plant Cell 21:985-999.

Pré, M., Atallah, M., Champion, A., De Vos, M., Pieterse, C. M., and Memelink, J. 2008. The AP2/ERF domain transcription factor ORA59 integrates jasmonic acid and ethylene signals in plant defense. Plant Physiol. 147:1347-1357.

Pruneda-Paz, J. L., Breton, G., Nagel, D. H., Kang, S. E., Bonaldi, K., Doherty, C. J., Ravelo, S., Galli, M., Ecker, J. R., and Kay, S. A. 2014. A genome-scale resource for the functional characterization of Arabidopsis transcription factors. Cell Rep. 8:622-632.

Ralhan, A., Schöttle, S., Thurow, C., Iven, T., Feussner, I., Polle, A., and Gatz, C. 2012. The vascular pathogen Verticillium longisporum requires a jasmonic acid-independent COI1 function in roots to elicit disease symptoms in Arabidopsis shoots. Plant Physiol. 159: 1192-1203.

Ramirez, S. R., and Basu, C. 2009. Comparative analyses of plant transcription factor databases. Curr. Genomics 10:10-17.

Reusche, M., Klásková, J., Thole, K., Truskina, J., Novák, O., Janz, D., Strnad, M., Spíchal, L., Lipka, V., and Teichmann, T. 2013. Stabilization of cytokinin levels enhances Arabidopsis resistance against Verticillium longisporum. Mol. Plant-Microbe Interact 26:850-860.

Sanchez-Vallet, A., Ramos, B., Bednarek, P., López, G., PiślewskaBednarek, M., Schulze-Lefert, P., and Molina, A. 2010. Tryptophanderived secondary metabolites in Arabidopsis thaliana confer non-host resistance to necrotrophic Plectosphaerella cucumerina fungi. Plant J. 63:115-127.

Schuhegger, R., Nafisi, M., Mansourova, M., Petersen, B. L., Olsen, C. E., Svatos, A., Halkier, B. A., and Glawischnig, E. 2006. CYP71B15 (PAD3) catalyzes the final step in camalexin biosynthesis. Plant Physiol. 141:1248-1254.

Schwab, R., Ossowski, S., Riester, M., Warthmann, N., and Weigel, D. 2006. Highly specific gene silencing by artificial microRNAs in Arabidopsis. Plant Cell 18:1121-1133.

Son, G. H., Wan, J., Kim, H. J., Nguyen, X. C., Chung, W. S., Hong, J. C., and Stacey, G. 2012. Ethylene-responsive element-binding factor 5, ERF5, is involved in chitin-induced innate immunity response. Mol. Plant-Microbe Interact 25:48-60.

Walper, E., Weiste, C., Mueller, M. J., Hamberg, M., and Dröge-Laser, W. 2016. Screen identifying Arabidopsis transcription factors involved in the response to 9-lipoxygenase-derived oxylipins. PLoS One 11: e0153216.

Wang, X., Liu, S., Tian, H., Wang, S., and Chen, J.-G. 2015. The small ethylene response factor ERF96 is involved in the regulation of the abscisic acid response in Arabidopsis. Front. Plant Sci. 6:1064.

Wehner, N., Hartmann, L., Ehlert, A., Böttner, S., Oñate-Sánchez, L., and Dröge-Laser, W. 2011a. High-throughput protoplast transactivation (PTA) system for the analysis of Arabidopsis transcription factor function. Plant J. 68:560-569.

Wehner, N., Weiste, C., and Dröge-Laser, W. 2011b. Molecular screening tools to study Arabidopsis transcription factors. Front. Plant Sci. 2:68.

Weigel, R., and Glazebrook, J. 2002. Arabidopsis: A Laboratory Manual. Cold Spring Harbour Laboratory Press, New York. 
Weiste, C., and Dröge-Laser, W. 2014. The Arabidopsis transcription factor bZIP11 activates auxin-mediated transcription by recruiting the histone acetylation machinery. Nat. Commun. 5:3883.

Weiste, C., Iven, T., Fischer, U., Oñate-Sánchez, L., and Dröge-Laser, W. 2007. In planta ORFeome analysis by large-scale over-expression of GATEWAY-compatible cDNA clones: Screening of ERF transcription factors involved in abiotic stress defense. Plant J. 52:382-390.

Xu, J., Meng, J., Meng, X., Zhao, Y., Liu, J., Sun, T., Liu, Y., Wang, Q., and Zhang, S. 2016. Pathogen-responsive MPK3 and MPK6 reprogram the biosynthesis of indole glucosinolates and their derivatives in Arabidopsis immunity. Plant Cell 28:1144-1162.

Yilmaz, A., Mejia-Guerra, M. K., Kurz, K., Liang, X., Welch, L., and Grotewold, E. 2011. AGRIS: The Arabidopsis Gene Regulatory Information Server, an update. Nucleic Acids Res. 39:D1118-D1122.
Zander, M., Thurow, C., and Gatz, C. 2014. TGA transcription factors activate the salicylic acid-suppressible branch of the ethylene-induced defense program by regulating ORA59 expression. Plant Physiol. 165:1671-1683.

Zarei, A., Körbes, A. P., Younessi, P., Montiel, G., Champion, A., and Memelink, J. 2011. Two GCC boxes and AP2/ERF-domain transcription factor ORA59 in jasmonate/ethylene-mediated activation of the PDF1.2 promoter in Arabidopsis. Plant Mol. Biol. 75:321-331.

Zeise, K., and von Tiedemann, A. 2002. Host specialization among vegetative compatibility groups of Verticillium dahliae in relation to Verticillium longisporum. J. Phytopathol. 150:112-119.

Zhao, Y., Hull, A. K., Gupta, N. R., Goss, K. A., Alonso, J., Ecker, J. R., Normanly, J., Chory, J., and Celenza, J. L. 2002. Trp-dependent auxin biosynthesis in Arabidopsis: Involvement of cytochrome P450s CYP79B2 and CYP79B3. Genes Dev. 16:3100-3112. 